bioRxiv preprint doi: https://doi.org/10.1101/2020.05.14.096024; this version posted May 19, 2020. The copyright holder for this preprint (which

was not certified by peer review) is the author/funder, who has granted bioRxiv a license to display the preprint in perpetuity. It is made available under aCC-BY-NC-ND 4.0 International license.

\title{
Recurrent evolution of two competing haplotypes in an insect DNA virus
}

\section{Tom Hill ${ }^{1 *}$, Robert L. Unckless ${ }^{1}$}

1. 4055 Haworth Hall, The Department of Molecular Biosciences, University of Kansas, 1200 Sunnyside Avenue, Lawrence, KS 66045.

*Corresponding author - Email: tom.hill@ku.edu

Keywords: nudivirus, Drosophila innubila, DiNV, virulence, coevolution 


\section{Abstract}

2 Hosts and viruses are constantly evolving in response to each other: as hosts attempt to suppress the virus,

3 the virus attempts to evade and suppress the host's immune system. This arms race results in the evolution

4 of novel pathways in both the host and virus to gain the upper hand. Here we describe the coevolution

5 between Drosophila species and a common and virulent DNA virus. We identify two distinct viral types

6 that differ 100-fold in viral titer in infected individuals, with similar effects across multiple species. Our

7 analysis suggests that one of the viral types appears to have recurrently evolved at least 4 times in the past

$8 \sim 30,000$ years, including in another geographically distinct species, due to the high effective mutation rate

9 which increases with titer. The higher titer viral type is associated with suppression of the host immune

10 system and an increased transmission rate compared to the low viral titer type. Both types are maintained

11 in all populations, likely due to an increased virulence in the high titer type creating a trade-off between

12 effective transmission and virulence and resulting in nearly equal reproduction rates $\left(\mathrm{R}_{0}\right)$ in both types.

13 Together these results suggest that the reciprocal selective pressures caused by the co-evolution between

14 host and virus has resulted in this recurrently evolving relationship.

\section{Introduction}

16 Antagonistic coevolution between hosts and their parasites is nearly ubiquitous across the tree of life. As a

17 result, genes involved in immune defense are among the fastest evolving genes in host genomes (NIELSEN

18 et al. 2005; SACKTON et al. 2007; ENARD et al. 2016; SHULTZ AND SACKTON 2019). Viruses are a particular

19 fitness burden on hosts; for viruses to persist within populations, they must successfully invade the host

20 organism, contend with the host immune system, replicate and then transmit the newly produced particles

21 to a new host (HOLMES 2007; GIFFORD 2012). Once successfully established in a population, natural

22 selection acts to modulate the rate the virus propagates relative to its virulence, optimizing the ratio of viral

23 virulence to transmission (WILLIAMS AND NESSE 1991; MAY AND NOWAK 1995; LIPSITCH et al. 1996).

24 Thanks to their elevated mutation rate and high population sizes, viruses can easily do all of this, and even

25 co-opt or manipulate host-pathways in the process (BURGYAN AND HAVELDA 2011; DAVEY et al. 2011;

26 PALMER et al. 2019). As a result, proteins that interact with viruses often show the fastest rates of evolution,

27 and the highest rates of adaptation compared to the rest of the genome, as the host attempts to suppress the

28 pathogen, or escape infection (OBBARD et al. 2006; OBBARD et al. 2009a; MUKHERJEE et al. 2013; ENARD

29 et al. 2016; PALMER et al. 2018a).

30 Hosts have evolved numerous immune pathways to reduce viral burden and enhance survival after

31 infection (MERKLING AND VAN RIJ 2013; WEST AND SILVERMAN 2018). Interestingly, several of these

32 pathways (IMD, Toll, JAK-STAT) are generally associated with other pathogens, yet also respond to

33 infection by viruses, though the specific mechanisms are not yet known (HOFFMANN 2003; HULTMARK 
2003; COCCIA et al. 2004; ZAMBON et al. 2005; COSTA et al. 2009; FERREIRA et al. 2014; WEST AND SILVERMAN 2018). In Drosophila melanogaster, the RNA interference (RNAi) pathway is involved in resisting viral infection by generating suppressive RNAs complementary to the viral sequence (HULTMARK 2003). As might be expected, this antiviral RNAi pathway is also rapidly evolving in many species (OBBARD et al. 2006; WANG et al. 2006; OBBARD et al. 2009b; MERKLING AND VAN RIJ 2013).

Drosophila innubila Nudivirus (DiNV), which infects the host D. innubila, is the one of the few DNA viruses to naturally infect Drosophila, and the only documented case of a DNA virus infection at high frequency in natural Drosophila populations (UNCKLESS 2011; HILl et al. 2019). Drosophila innubila is a mushroom feeding species of the Drosophila subgenus found inhabiting woodlands found on mountains across Arizona \& New Mexico, separated by large expanses of desert. D. innubila radiated north from Mexico during the last glaciation period and came to inhabit these forests after the glacial retreat, creating a subdivided population with high rates of gene flow between locations (DYER AND JAENIKE 2005; HILL AND UNCKLESS 2020).

During this period D. innubila likely became infected with DiNV, suggesting a long-lasting hostpathogen relationship in multiple populations. This could lead to opportunities to study the coevolution of DiNV and D. innubila in replicate (HILL AND UNCKLESS 2020), which could potentially result in parallel or divergent evolution of the virus and interacting host pathways (ANDERSON AND MAY 1982; KALTZ AND SHYKOFF 1998).

A pair of previous studies examined the rates of evolution of DiNV and D. innubila, finding the envelope and replication machinery to be rapidly evolving in DiNV, suggesting its importance in viral propagation (HILL AND UNCKLESS 2018). In D. innubila, the antiviral RNAi machinery is not rapidly evolving, possibly as DNA viruses interact with different immune pathways to RNA viruses (the primary burden of D. melanogaster) (WEBSTER et al. 2015). Consistent with this, the Toll pathway is both rapidly evolving in D. innubila and suppressed by a related nudivirus upon infection in D. melanogaster (HILL et al. 2019; PALMER et al. 2019).

DNA viruses, and nudiviruses such as DiNV in particular, have much larger genomes (100kbp or larger) than RNA viruses, with much more complicated replication cycles (ROHRMANN 2013). RNA viruses also have much higher mutation rates than DNA viruses, yet much lower levels of diversity due to lower recombination rates and efficient selection on variation in the genome (PENNINGS 2012; PENNINGS et al. 2014; WILSON et al. 2016; FEDER et al. 2019). As a result, we expect the evolutionary dynamics of DNA viruses to differ dramatically from RNA viruses (ROHRMANN 2013; HILL AND UNCKLESS 2017). In fact, because of their large genomes, high recombination rate and low mutation rate, we expect that DNA virus coevolution with hosts will be qualitatively different than RNA viruses or bacteria. Together this paints a

67 picture suggesting that hosts can have different relationships with different pathogens (such as RNA viruses 
or DNA viruses), and the pathogens themselves can behave differently within the host. Characterizing the relationships between different species and their long-term pathogens, such as DiNV and D. innubila, will help broaden and expand our understanding of how hosts and pathogens evolve in response to each other.

Here, we survey the genetic variation in DiNV to infer its co-evolutionary history with D. innubila and two other associated hosts. We identify two viral multilocus genotypes (considered to be haplotypes) that differ by 11 focal SNPs and show that these viral types are maintained within the same host population and across multiple isolated host populations. Despite high rates of recombination, these SNPs are tightly linked likely due to extremely strong selection and possibly incompatibilities between types. One viral type is associated with 100-fold higher viral titer and increased virulence compared to the other. Further, we find evidence that the high titer type evolved independently in at least four geographically-isolated host populations. Together, these results suggest rapid evolutionary dynamics of host-virus interactions, due to the multiple competing viral types that interact with different host pathways.

\section{Results}

DiNV segregates for linked variants strongly associated with viral titer

82 To characterize the evolutionary dynamics of wild Drosophila innubila Nudivirus (DiNV) in its host (D.

83 innubila), we sequenced wild-caught individuals from four populations with the expectation that some 84 ( $40 \%$ in previous samples) individuals would be infected (HILL AND UNCKLESS 2020). We considered strains to be infected with DiNV if they had at least $10 \mathrm{x}$ coverage for $95 \%$ of the genome. In total, we used sequencing information for 57, 92, 92, and 92 individuals from the Huachucas (HU), Santa Ritas (SR), Chiricahuas $(\mathrm{CH})$, and Prescott (PR) populations with infection rates 26\%,44\%, 63\% and 79\%, respectively (Supplementary Table 1). We also used 35 individuals collected in the Chiricahuas in 2001 (52\% infected with DiNV) and 80 individuals collected in the Chiricahua's in 2018 (Supplementary Table 2, pre-selected using PCR, 40 infected with DiNV and 40 uninfected).

We isolated and sequenced DNA from these samples and, after filtering and mapping to the genome

92 we called variation in the viral genomes to assess the extent of adaptation in each viral population.

93 Consistent with an arms race between host and virus, most envelope and novel virulence (GrBNV-like)

94 genes show strong signatures of adaptive evolution in each population compared to background viral genes

95 (using McDonald-Kreitman based statistic Direction of Selection (STOLETZKI AND EYRE-WALKER 2011)

96 and Selection Effect (EILERTSON et al. 2012), Supplementary Figure 1, DoS > 0, GLM $p$-value < 0.05).

Given these potential signatures of an arms race between $D$. innubila and DINV, we first attempted

98 to determine if any host or viral genetic variation is associated with within-host viral titer, which we use

99 here as a measure of virulence. For each virus-infected individual, we quantified viral titer (as viral genome

100 coverage normalized to host autosomal genome coverage) and identified both host and viral 
101 polymorphisms. We then performed an association study across both host and virus variable sites to identify variants significantly associated with viral titer using PLINK (PURCELL et al. 2007).

Of 5,283 viral SNPs in the 155kbp DiNV genome, 1,403 SNPs are segregating in at least 5 infected host individuals. Of those 1,403 SNPs, 78 are significantly associated with viral titer after multiple testing correction (FDR $<0.01$, Figure 1A). Of these, 16 are within 2000bp of the start site of a gene, 18 are coding nonsynonymous, 11 are coding synonymous and 33 are intergenic. The most significantly associated SNP

107 is a non-synonymous polymorphism in the active site of Helicase-2 (Figure 1A, Supplementary Figure 2).

108 The Helicase-2 polymorphism is the only significantly associated polymorphism found segregating at a 109 range of frequencies within individuals (Supplementary Figure 2). The frequency of this derived 110 polymorphism has a negative relationship between viral titer and the derived SNPs frequency (GLM t-value $=-20.516, p$-value $=5.55 \mathrm{e}-62$ ). However, when ranking samples by viral titer, the SNP frequency does not

112 fit this expectation and several samples fixed for the ancestral allele also have a lower viral titer 113 (Supplementary Figure 2).

114 We also identified a striking association between viral titer and eleven nearly perfectly linked 115 polymorphisms found across the DiNV genome (Figure 1A, highlighted SNPs, Table 1, Supplementary

116 Figures 2-4, Sig. SNPs). We refer to these two types as the 'High Type' and 'Low Type' (Figure 1B).

117 This multilocus genotype includes three non-synonymous SNPs and five SNPs in the UTRs of known

118 virulence factor genes and three intergenic SNPs. Viral titer is, on average, 100-fold higher in individuals 119 infected with High type virus compared to the ancestral Low type (Figure 1B). Though we found few 120 strains with an intermediate number of SNPs, viral titer increases as the number of High type SNPs 121 increases (Figure 1C, GLM t-value $=34.971, p$-value $=5.912 \mathrm{e}-16)$, though the rate of increase slows as 122 the number of High type SNPs increases suggesting diminishing returns (Figure 1C). Some of these 123 polymorphisms are associated with known virulence factors, or are related to the formation of the viral 124 envelope co-opting the host vesicle trafficking system and are rapidly evolving in nudiviruses (e.g. VLF125 1, ODV-E56, PIF-3) (RoHRMANN 2013; HiLl AND UNCKLESS 2017; HiLl AND UNCKLESS 2018).

126 Additionally, several are associated with orphan genes thought to be novel virulence factors, including a

127 gp83, a gene that downregulates Toll-induced antimicrobial peptides (AMPs) and upregulates those 128 induced by IMD (gp83) (PALMER et al. 2019). Both pathways may interact with DNA viruses (ZAMBON 129 et al. 2005; COSTA et al. 2009; MERKLING AND VAN RIJ 2013; FERREIRA et al. 2014; LAMIABLE et al. 130 2016; PALMER et al. 2019).

131 Among populations there is a positive correlation between the frequency of the High type and 132 overall DiNV infection frequency (Figure 1D, GLM logistic regression z-value $=6.104, p$-value $=$ 133 0.00883), suggesting that the High type may have a higher effective transmission rate, resulting in a 134 higher number of new individuals infected, per DiNV infected individual. The transmission rate appears 
135 to be higher for the High type, despite a possibly higher death rate than the Low type. We also find both

136 viral types in collections from 2001 and 2017, with the High type significantly more common in the 2017

137 collection (Fisher Exact Test $p$-value $=0.0167$, Figure 1D).

138 Table 1: Candidate viral SNPs associated with viral titer, their loci, associated genes and the functional 139 category of that gene.

\begin{tabular}{|c|c|c|c|}
\hline SNP loci & Nearest gene & SNP functional annotation & Nearest gene functional annotation \\
\hline G14249T & $V L F-1$ & Non-Synonymous & $\begin{array}{l}\text { Required for late stage virus activity } \\
\text { and particle assembly }\end{array}$ \\
\hline $\mathrm{C} 41210 \mathrm{~T}$ & $L E F-4$ & Non-Synonymous & $\begin{array}{l}\text { RNA polymerase subunit for RNA } \\
\text { modification }\end{array}$ \\
\hline G42389T & gp 83 & Upstream & $\begin{array}{l}\text { Suspected virulence factor which } \\
\text { suppresses Toll activity }\end{array}$ \\
\hline A59194G & gp51 & Intergenic & Suspected virulence factor \\
\hline C59275A & $P I F-6$ & Upstream & $\begin{array}{l}\text { Per OS Infectivity factor envelope } \\
\text { protein, required for oral infection }\end{array}$ \\
\hline T59276C & $P I F-6$ & Upstream & $\begin{array}{l}\text { Per OS Infectivity factor envelope } \\
\text { protein, required for oral infection }\end{array}$ \\
\hline G66615A & gp19 & Non-Synonymous & Suspected virulence factor \\
\hline $\mathrm{C} 78978 \mathrm{~T}$ & gp94 & Intergenic & Suspected virulence factor \\
\hline G78991A & gp94 & Intergenic & Suspected virulence factor \\
\hline C126118A & $O D V-E 56-2$ & Upstream & $\begin{array}{l}\text { Occlusion-derived virus envelope } \\
\text { protein required for particle formation }\end{array}$ \\
\hline A132593C & Helicase-2 & Non-Synonymous & $\begin{array}{c}\text { Unwinds DNA and is critical for DNA } \\
\text { replication }\end{array}$ \\
\hline $\mathrm{T} 140117 \mathrm{C}$ & $P I F-3$ & Upstream & $\begin{array}{l}\text { Per OS Infectivity factor envelope } \\
\text { protein, required for oral infection }\end{array}$ \\
\hline
\end{tabular}

Further, the expression of the virally encoded suppressor of Toll signaling, gp83, per viral particle

142 is greater in strains containing at least one derived SNP of the high type increase (Figure 1E, GLM t-value

$143=10.32, p$-value $=7.34 \mathrm{e}-12$ ), suggesting enhanced virulence in the high type. For these SNPs, few are

144 found at intermediate frequencies within samples (Supplementary Figure 2), and no samples contain more

145 than two SNPs at a similar frequency (Supplementary Figure 2). This suggests that hosts are either

146 infected completely with Low type or High type virus particles and the two types may be partially 
147 incompatible with each other. Together, these results suggest that the high viral type is more virulent

148 possibly because it is better able to suppress host Toll signaling.

149 Figure 1: Viral genome-wide association study for DiNV titer in wild D. innubila. A. Manhattan plot for

150 each DiNV SNP and the significance of its association with DiNV titer. SNPs are colored if they are

151 upstream, synonymous, non-synonymous or nonsense mutations. 12 named SNPs are either part of the

152 significantly associated viral haplotype or are in Helicase-2. The FDR corrected $p$-value cutoff of 0.01 is

153 shown as a dashed line (multiple testing correction for 1,403 tests). B. Viral titer for individual wild

154 caught flies infected with Low and High DiNV haplotypes (containing all high-type SNPs). The middle

155 bar represents median value, upper and lower bars represent $25^{\text {th }}$ and $75^{\text {th }}$ percentile and whiskers

156 represent a 95\% confidence interval. C. Association between the number of significant SNPs and the viral

157 titer of a sample. D. Across five populations, the frequency of the High DiNV haplotype is correlated with

158 the frequency of the virus infection. E. Expression (in FPKM per viral particle) of $g p 83$ increases with the 159 number of High DiNV haplotype SNPs.
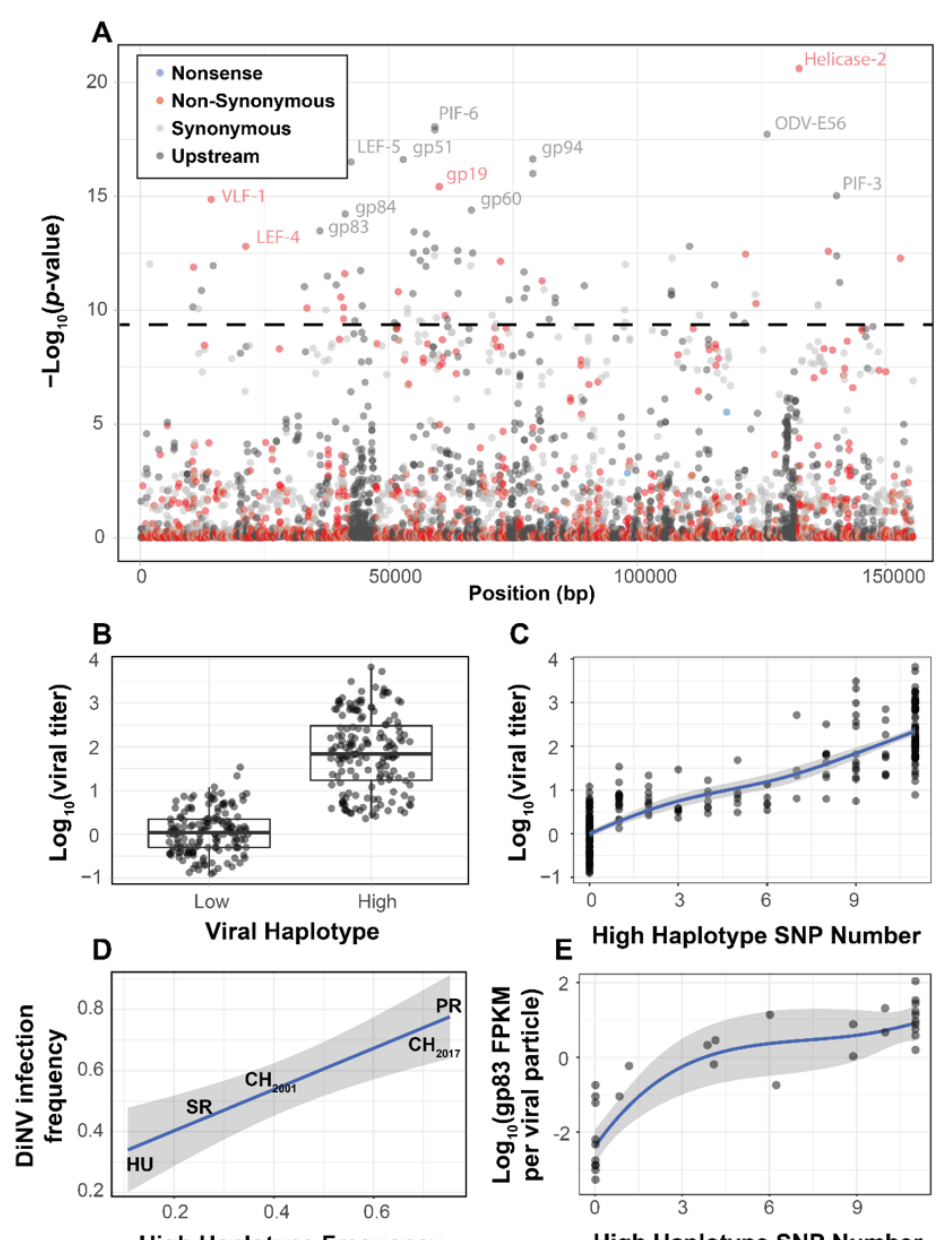
162 Given the striking difference in viral titer and infection frequencies across populations between High and

163 Low type viruses, we sought to further characterize the differences in infection dynamics between the types.

164 We sequenced mRNA from 80 wild D. innubila males collected in 2018 (Supplementary Table 2, 40

165 infected with DiNV, 40 uninfected) and performed a differential expression analysis between infected and

166 uninfected individuals. Few genes were differentially expressed (DE) between infection states in $D$. 167 innubila (Supplementary Figure 5A, $p$-value $<0.01$ after FDR multiple testing correction, above the dotted 168 line), but these DE genes were enriched for several interesting categories. Specifically, we found IMD169 induced antimicrobial peptides (AMPs) were upregulated upon DiNV infection, while one Toll-induced 170 AMP, and several chorion genes and heat shock proteins were downregulated (Supplementary Figure 5A). 171 We also compared these results to a laboratory experiment in D. melanogaster of differential expression 172 after infection with a close relative of DiNV (PALMER et al. 2018b). These same genes are also differentially 173 expressed in D. melanogaster. Of the 12 genes which are differentially expressed in the same direction in 174 both species, five are AMPs and five are chorion genes (Supplementary Figure 5B).

We then compared gene expression between $D$. innubila infected with High or Low type. We find $17 \mathrm{DE}$ host genes and $9 \mathrm{DE}$ viral genes between types (after controlling for virus copy number as FPKM/titer, Figure 2, FDR corrected $p$-value $<0.01$, GLM t-value $=-4.6239413, p$-value $=9.876143 \mathrm{e}-05$ ). Specifically, four Toll-mediated immune peptides (Listericin, IM33, Bomanins BomBC2 and BomT2) have reduced expression in High Type infected individuals compared to the Low Type (Figure 2, Supplementary

180 Figure 6). Finally, viral genes of interest ( $P I F-3, V L F-1, g p 83)$ have higher expression per viral particle 181 (FPKM/titer) in the High type compared to the Low type, all also increase in expression per viral particle 182 (FPKM/titer) as the number of High type alleles increases (Figure 1E \& 2, t-value = 13.732, $p$-value 3.36e183 15). This pattern may be driven by the allele of the non-synonymous SNP in $V L F-1$ (GLM t-value $=2.13$, $184 p$-value $=0.04272)$ and the alleles of the SNPs upstream of $g p 83, P I F-3, g p 51$ and $O D V$-E56 (GLM t-value $185=3.518, p$-value $=0.00162$ ). Together these results suggest that the high viral type has increased expression 186 of key virulence factors, which in turn, manipulate the expression of host genes involved in immune defense 187 to result in the observed differences in viral titer. These results suggest that higher gp83 expression may 188 cause the lower Toll-mediated AMP expression, possibly due to lowering Myd88 expression, which in turn 189 prevents the host from enacting a proper immune response to DiNV infection (Figure 2, Supplementary 190 Figure 5).

\section{Experimental infections recapitulate differences in viral type virulence}

192 To assess if the virulence of the virus types differs in experimental infections, we performed experimental 193 infections of D. innubila males using viral filtrate of strains infected with one of the two types of DiNV. 
194 As infectious viral titer is increased, survival decreases regardless of viral type (Supplementary Figure 7 \&

1958 , ANOVA residual deviance $=3.536, p$-value $=2.454 \mathrm{e}-07$, Cox Hazard Ratio z-value $>2.227, p$-value $<$

196 0.02592), with survival decreasing as titer increases (Supplementary Figure 7, Cox Hazard Ratio z-value >

$1975.428, p$-value $<5.69 \mathrm{e}-08$ ). In both cases viral titer also increases for the first 3 days of infection (GLM t-

198 value $=9.817, p$-value $=3.6 \mathrm{e}-14)$.

199

Figure 2: Differential expression of $D$. innubila and DiNV genes between $D$. innubila infected with either the Low type or High type DiNV multilocus genotypes. For host genes, the log-fold change of mRNA

202 fragments per million fragments is compared, while for viral genes the log-fold change of viral mRNA

203 fragments per million fragments per viral particle is compared. Genes are colored/labelled by categories of 204 interest, specifically antimicrobial peptides (AMPs), proteins involved in the extracellular matrix and viral 205 proteins. Specific genes of interest, such as Myd88, are also named. The FDR-correct significance cut-off of 0.01 (10,320 tests) is shown as a dashed line.

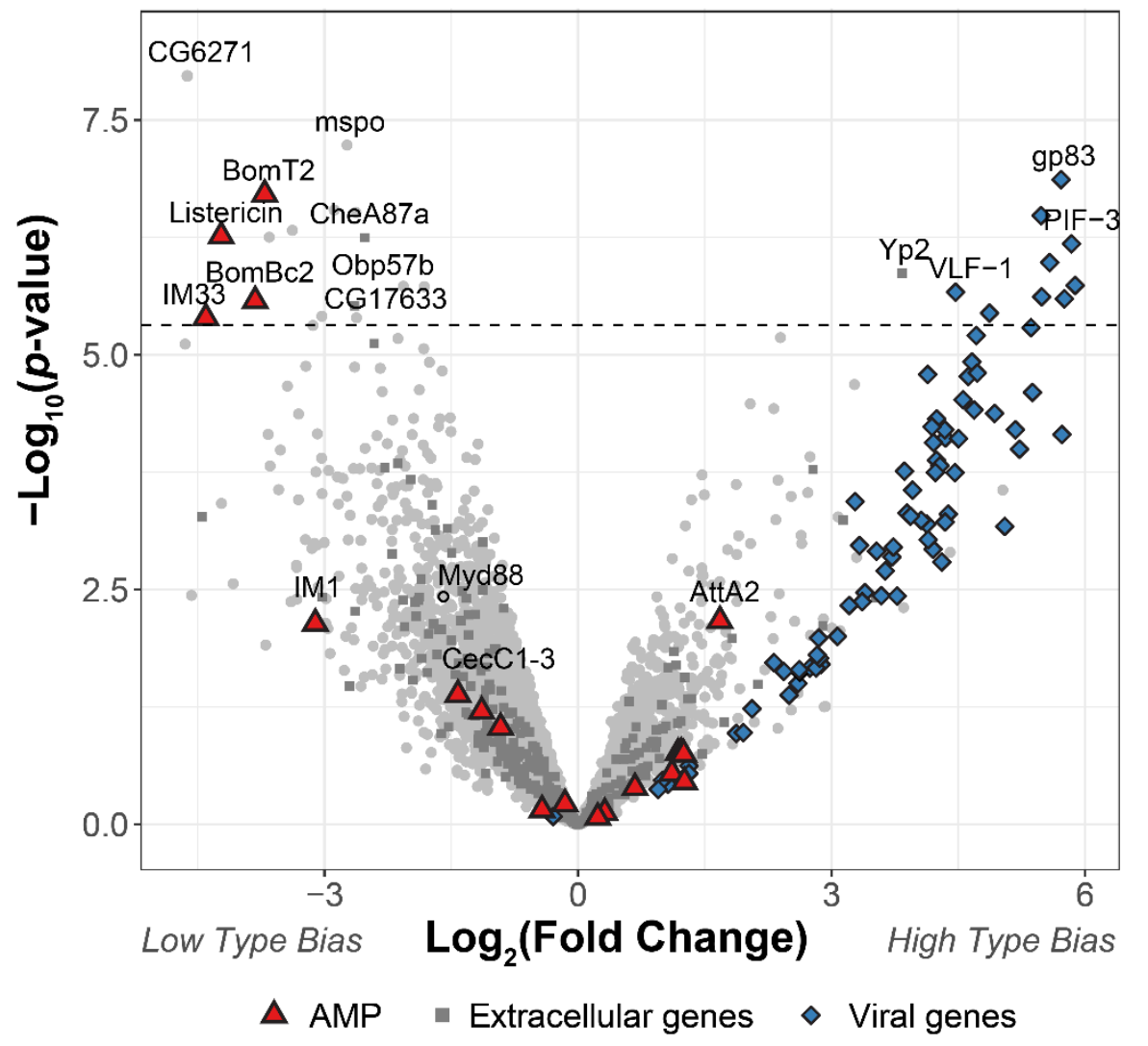

For a set of independent viral isolates, four High type- and four Low type-infected samples, we 
210 to filtration) and performed infections for replicates of 10 males with microneedles dipped in one of the

211 filtrate samples. Survival is significantly lower for flies infected with High type viruses when compared to

212 either flies pricked with sterile media (Figure 3A, Cox Hazard Ratio z-value $=3.671 p$-value $=0.000242$ )

213 or those pricked with Low type virus (Figure 3A, Cox Hazard Ratio z-value $=4.611 p$-value $=4 \mathrm{e}-06$ ). Flies

214 pricked with Low type virus do not show a significant reduction in survival compared to control flies (Cox

215 Hazard Ratio z-value $=1.353, p$-value $=0.176)$. We also measured viral titer over time using qPCR, and

216 find titer increases through time in flies infected with either type (Figure 3B, GLM $\log _{10}($ titer) $\sim$ days +

217 type, days t-value $=9.912, p$-value $=1.76 \mathrm{e}-14)$. Flies infected with High type virus have significantly higher

218 viral titer compared to flies infected with Low type virus (Figure 3B, GLM $\log _{10}($ titer) $\sim$ days + type, type

219 t-value $=3.934, p$-value $=0.000211$ ). These results suggest that while the High type strain has higher viral

220 titer and potentially higher transmission rate in wild flies, it also has higher virulence, even after controlling

221 for initial infection titer.

222 Figure 3: Effect of viral type in experimental infections. A. Survival curves of $D$. innubila infected with 223 high and low viral types compared to control flies pricked with sterile media, for 15 days post infection.

224 Survival 5 days post-infection separated by strain is shown in Supplementary Figure 8. B. qPCR copy 225 number of viral $p 47$ relative to tpi in D. innubila infected with DiNV filtrate of high and low types.
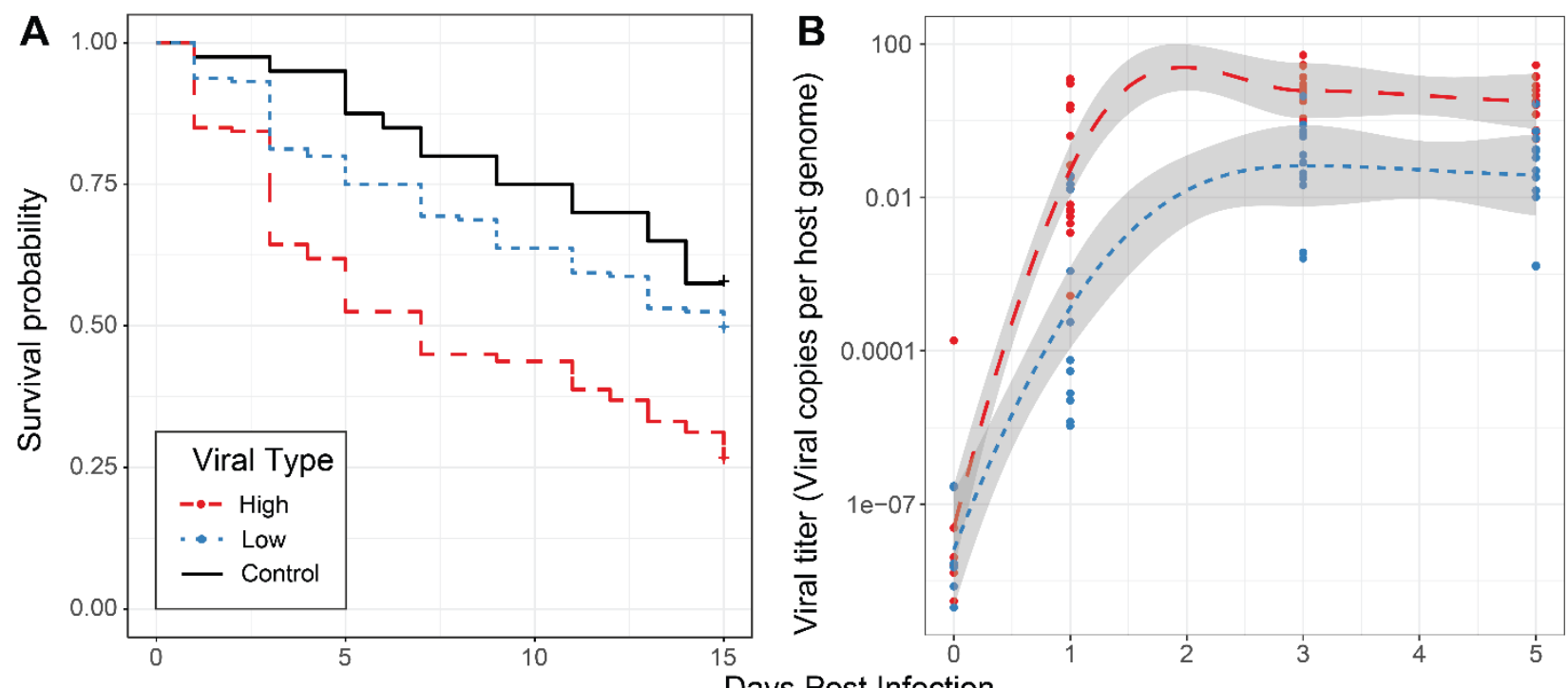

227 DiNV types are under strong selection in the host

228 Recombination is required during nudivirus replication and recombination start sites can be at any point

229 in the single chromosome circular genome (KELLY 1982; ROHRMANN 2013). These factors likely cause

230 the incredibly high recombination rates observed in nudiviruses (BLISSARD AND ROHRMANN 1990;

231 WANG AND Jehle 2009; Rohrmann 2013). In DiNV, the eleven key SNPs that distinguish the High and

232 Low haplotypes are spread across the genome yet are nearly perfectly linked. In contrast, other SNPs in 
the genome have relatively low linkage disequilibrium, suggesting that selection to maintain each haplotype is strong (Supplementary Figure 4 and 9).

Using McDonald-Kreitman based statistics for detecting selection (MCDONALD AND KREITMAN 1991; STOLETZKI AND EYRE-WALKER 2011; EILERTSON et al. 2012), we tested whether genes that are associated with the High and Low haplotypes exhibited different signatures of natural selection compared to other viral genes. Envelope and virulence proteins show significantly elevated signatures of adaptation (Figure 4, envelope $\&$ virulence versus background paired T-test t-value $=2.1761, p$-value $=0.03814$ ).

240 Genes found in the initial GWAS for virulence, which defined the High and Low types (such as VLF-1, 241 PIF-3, LEF-4 and LEF-5) have significantly higher rate of substitutions being fixed due to selection than 242 background genes (Figure 4, type-associated genes versus all other, t-value $=2.718, p$-value $=0.00068$ )

We also performed a GWAS using the host polymorphism and find few associated SNPs, after

244 controlling for the viral haplotype (Supplementary Figure 10). Consistent with the arms race model, host genes we suspect are interacting with DiNV (such as the GWAS hits, AMPs, chorion genes, piRNA genes and extracellular genes) show elevated levels of substitutions fixed by selection compared to background genes in D. innubila (Figure 4 \& Supplementary Figure 1). Finally, DE chorion genes, extracellular genes and AMPs have significantly more adaptive substitutions than similar non-DE genes (Figure 4, blue dots, differentially expressed versus all other T-test: $D$. innubila $\mathrm{t}$-value $=4.755, p$-value $=0.000671$ ). Overall these results suggest strong selection is acting on both the host to suppress viral activity and the virus to escape this suppression.

\section{The High viral type of DiNV evolved repeatedly in three D. innubila populations}

253 We next sought to understand the evolutionary origin of the two types. Given that both types are found in 254 all populations surveyed (Figure 1D), we hypothesized that this could occur one of three ways: First, the 255 derived haplotype was present ancestrally and has been maintained since before geographic isolation 256 occurred. Second, the derived haplotype evolved following geographic isolation and has spread via 257 migration between locations. Third, the derived haplotype has recurrently evolved in each location.

To distinguish between these possibilities and determine the timeframe of divergence, we used the site frequency spectrum of silent DiNV polymorphism to estimate effective population size backwards in 260 time for all populations (LIU AND FU 2015). We find three populations (CH, HU and SR) expand from a 261 single viral particle $\left(\mathrm{N}_{\mathrm{e}}=1\right)$ to millions of particles during the last glacial maximum (30-100 thousand years 262 ago) when D. innubila settled its current range (Supplementary Figure 11). This supports a single invasion 263 event during a host-range change. PR appears to expand between 1 and 10 thousand years ago, suggesting 264 a much more recent bottleneck during the range expansion in PR (Supplementary Figure 11) (HILL AND 265 UNCKLESS 2020). 
We aligned genomic regions containing SNPs to two related nudiviruses, Kallithea virus and Oryctes rhinoceros Nudivirus (OrNV) (WANG et al. 2008; HILL AND UNCKLESS 2018; PALMER et al. 2018b). The High haplotype alleles are not present in either Kallithea or OrNV, and are not found in short read information for wild D. melanogaster infected with Kallithea virus (WEBSTER et al. 2015), suggesting they are derived in DiNV.

Figure 4: Genes implicated in host/virus interaction are rapidly evolving by positive selection in the 273 Chiricahua population. Difference in selection effect for viral and host gene categories of interest, and 274 nearby background genes, as indicated by the proportion of substitutions fixed by adaptation, weighted by 275 mutations in SnIPRE (EILERTSON et al. 2012). Genes that have associated SNPs from the GWAS are 276 highlighted in red, while genes which are differentially expressed upon infection, or between viral types 277 are labelled in blue. All GWAS hits are also differentially expressed and labelled in red. Genes of interest 278 are named.

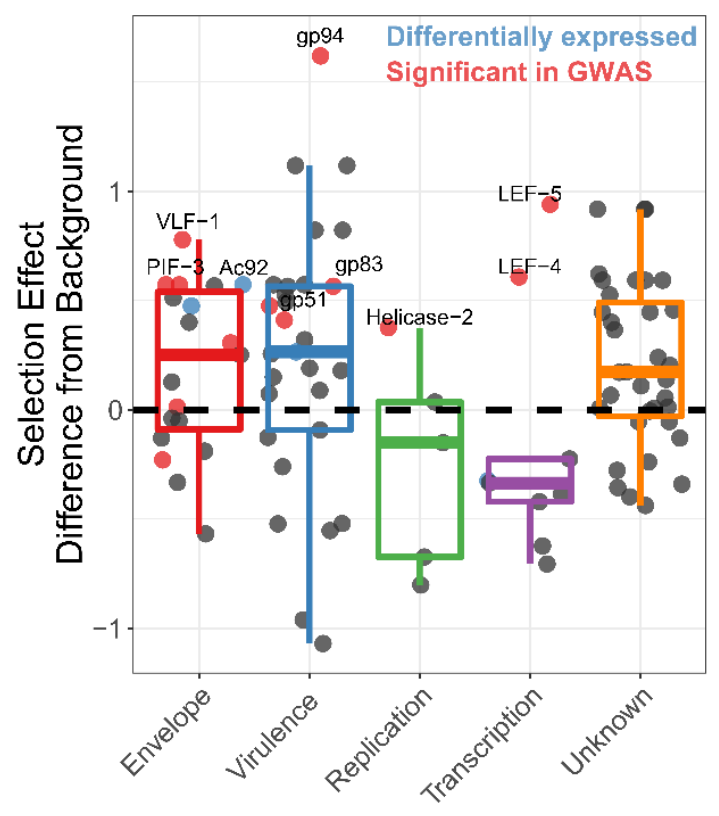

Viral Gene Category

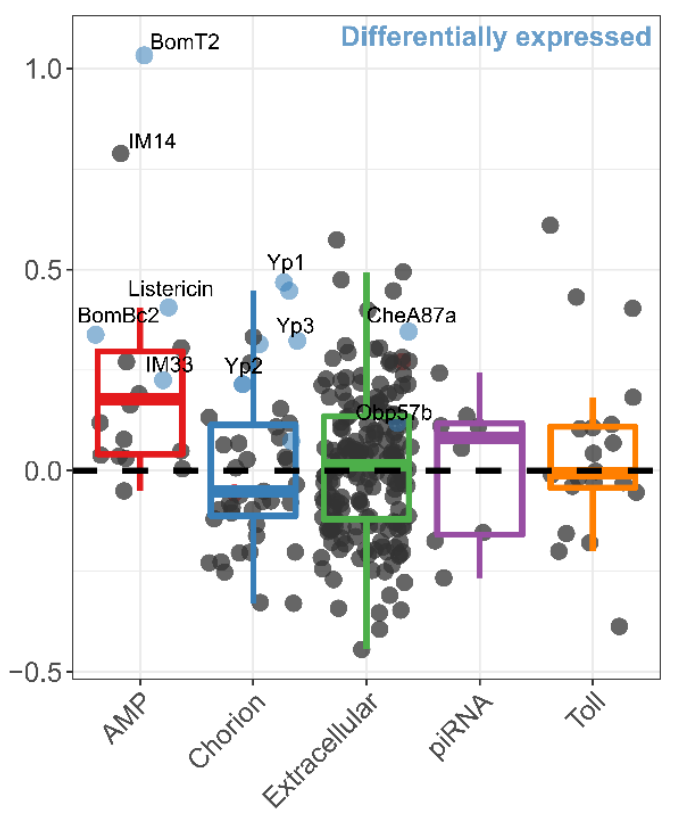

Host Gene Category

We generated consensus DiNV sequences for each infected $D$. innubila individual and created a whole genome phylogeny to infer geographic diffusion of samples using BEAST (BOUCKAERT et al. 2014).

282 We then performed ancestral reconstruction of the presence of the High type across the phylogeny using 283 APE (PARADIS et al. 2004). Our samples group as three populations (with HU and SR forming one 284 population) and, consistent with our expectation, the Low type is the ancestral state (Figure 5A). 285 Surprisingly, the High type appears to have evolved repeatedly and convergently within each population, 
forming separate groups within each population (Figure 5A). The High type also clusters within each population in a principal component analysis of all viral SNPs (Supplementary Figure 9), and when repeating these analyses while excluding the eleven focal SNPs.

We next surveyed each background SNP (not associated with the High or Low type) to determine if the general background supports one of the three outlined ways in which the High type evolved and spread in each location. We grouped SNPs by their presence in just the High type or Low type (supporting a single origin and spread by migration) or if they were unique to a single population but shared between the High and Low types (supporting recurrent evolution with recombination).

In total, 391 SNPs (28\% of SNPs surveyed) are unique to a single population yet are still shared between both High and Low types (Figure 5 and Supplementary Figure 9), compared to 23 SNPs shared between locations but exclusive to High type samples.

These unique SNPs (161 for CH, 127 for HU and SR, and 53 for PR), are present in all High type samples of a population but a variable proportion of Low types for that population (between 19\% and 94\%) and are unique to that population. This pattern fits with the High type recurrently evolving on a single background (a different background in each location), supporting recurrent evolution of the High type. The population-specific background SNPs are spread throughout the DiNV genome, with little evidence of recombination with the high type SNPs, making it unlikely that these SNPs recombined onto different backgrounds (Supplementary Figure 4).

Though there is strong linkage between the High type SNPs, they are not perfectly associated with each other (Supplementary Figure 4). Using this slight disassociation and APE (PARADIS et al. 2004), we performed ancestral reconstruction of SNP origins in each population (assuming recurrent evolution) and find that, excluding three variable SNPs, the evolution of these SNPs was the same order in each population (Figure 5B).

To determine if this recurrent evolution is plausible in our estimated timeframe $(\sim 10,000$ years $)$, we simulated viral populations using a modified discrete SIR model using deSolve (SOETAERT et al. 2010) with estimated baculovirus mutation rates, ranges of viral titer taken our samples and estimated population sizes for each viral population (parameters described in the methods). In this model we used an effective mutation rate scaled to viral titer, considering the mutation rate per particle, so total mutations per generation increase with viral titer. The simulations suggest that waiting time for the first mutation that increases titer is highly variable between replicates but usually occurs within 1000 generations ( 200 years assuming 5 generations per year, in $>99.1 \%$ of replicates, Figure $5 \mathrm{C}$ ). The average wait time for each subsequent mutation decreases monotonically $($ GLM t-value $=-2.389, p$-value $=0.03686)$. In each case, the next mutation appears in the background of the previous high titer mutation (Figure 5C) due to the elevated effective mutation rate and increased basic reproduction number $\left(\mathrm{R}_{0}\right)$. The accumulation of 
320 mutations therefore occurs at a geometric (approximately exponential) rate. Additionally, the standard

321 deviation of time wait times also decreases with each new mutation (GLM t-value $=-2.441, p$-value $=$

3220.04241 ), increasing the certainty that the entire multilocus genotype will appear in a population rapidly

323 once the initial mutations appear. This chain reaction of adaptation facilitates the repeated evolution of the

324 virulent High type independently in three populations, with all eleven mutations fixing in a population

325 within 6000 generations ( $\sim 200$ years) in all replicates (3372 generations on average, $\sim 675$ years), a

326 plausible amount of time given our estimated timeframe.

327 Both viral types are found in two other Drosophila species and have also evolved in a geographically 328 distinct population

329 Since we find two types of DiNV are maintained, and that other species are infected with DiNV, we 330 hypothesized that another species could be a reservoir for the less effective Low type. We chose to study 331 D. azteca from the Chiricahuas since it is frequently infected with DiNV ( $\sim 33 \%$ infection), overlaps with

332 D. innubila, and is genetically divergent (40-60 million years) which could mean a very different genetic 333 interaction between host and virus (UNCKLESS 2011). We also examined DiNV-infected D. falleni 334 (collected in Georgia) as an outgroup. In all, we sequenced 36 D. azteca and 56 D. falleni. Both types are 335 present in all examined species, but the high type is rare in D. azteca (Figure 6B). The High type has a 336 significantly higher titer than the low type in all cases (Figure 6A). Viral titer is not significantly different 337 across species for either High or Low type (Figure 6A, GLM t-value $=-1.351, p$-value $=0.179$ ). We also 338 find the D. azteca samples cluster with $\mathrm{CH}$ D. innubila samples and contain the $\mathrm{CH}$ background SNPs 339 (Supplementary Figure 9C), suggesting no differentiation in the virus infecting different species. 340 Interestingly, D. falleni DiNV clusters completely separately from the other samples, likely due to its 341 geographic separation, but still has a derived cluster of High type virus, suggesting a fourth separate 342 evolution of the High type in Georgia. Despite the lack of difference between species samples in Arizona, 343 a lower proportion of the D. azteca population is infected with DiNV, and the High Type is less common 344 than the Low type DiNV (Figure 6B). Thus perhaps, even though the relative differences in titer are 345 preserved between the two species, the Low Type is favored in D. azteca because this reduced virulence 346 leads to a greater $\mathrm{R}_{0}$ in $D$. azteca. Thus, the two types of the virus may be maintained in both host species 347 because though they have become specialized to maximize fitness in one host, messy transmission between 348 host species could lead to their continued presence in both hosts.

349 We also repeated the GWAS for viral titer in DiNV infecting D. azteca and D. falleni. In both cases 350 we again found the 11 High type SNPs associated with viral titer (GLM t-value $>4.28, p$-value $>0.0001$ in 351 both cases), but not the Helicase-2 SNP (despite its presence in D. falleni DiNV samples). After controlling 352 for the High type, we find no other significant DiNV SNPs in D. azteca associated with viral titer. For 
DiNV infecting D. falleni, we find 478 significant SNPs (FDR corrected $p$-value $<0.01$ ), though none of

354 them with as large an effect as the High type associated SNPs.

356 Figure 5: The evolution and maintenance of two viral types. A. Phylogeographic reconstruction of the spread of DiNV through D. innubila, rooted on the Kallithea virus reference sequence, including a

358 reconstruction of the High type evolution (with strains containing all 11 High type variants shown in red,

359 strains with an intermediate number of high type variants are shown in pink, and strains with no high type

360 variants are shown in black). Branches are colored when the SNPs found in the background for each High

361 haplotype are present in the population, showing that the background differs per population. Black

362 branches show states where branch tips do not contain all the shared High/Low population specific

363 background SNPs. B. Order of mutations in the viral haplotype appearing in each population. Apart from

364 three mutations the order is consistent between locations. C. The number of generations needed for 'High 365 titer' mutations to evolve in simulated populations, given that each mutation increases the mutation rate.

366 The number of generations between each mutation appearing decreases as titer increases.

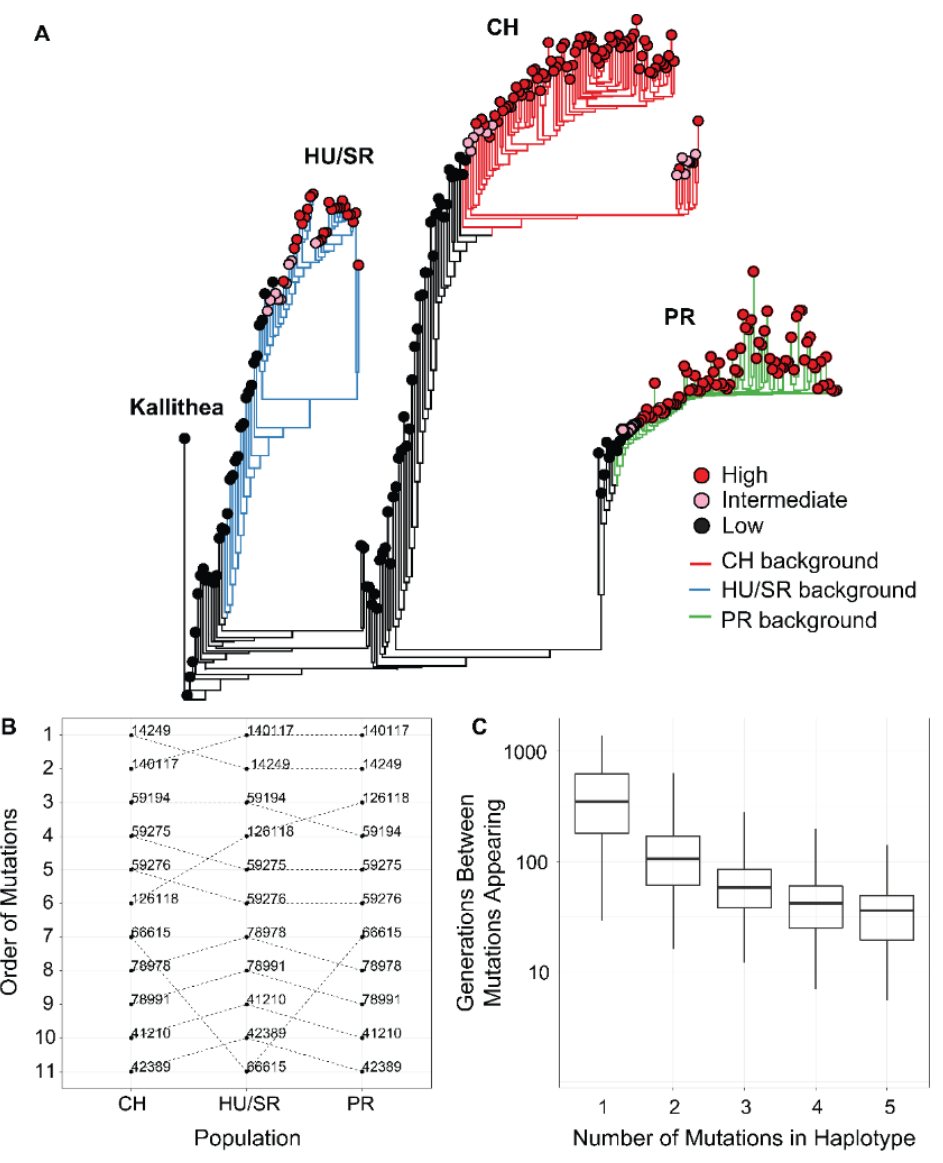


Figure 6: A. Viral titer for $\mathrm{CH}$ samples of D. azteca, D. falleni and D. innubila infected with High and Low type DiNV. B. Proportion of D. azteca and D. innubila $2017 \mathrm{CH}$ population infected with High and Low type DiNV.

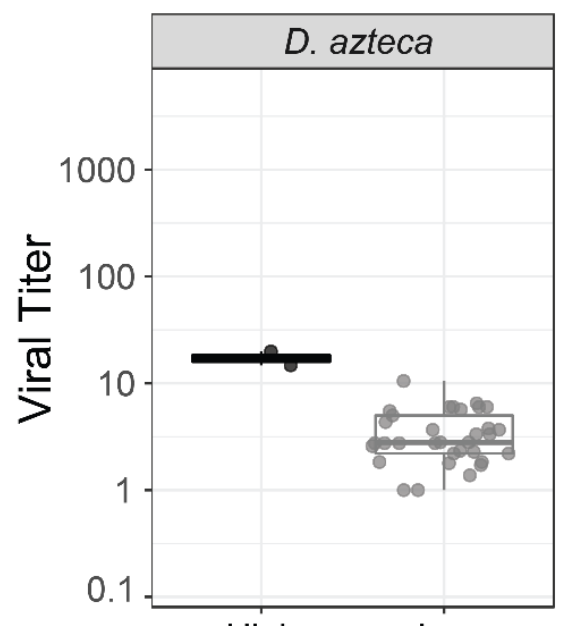

High
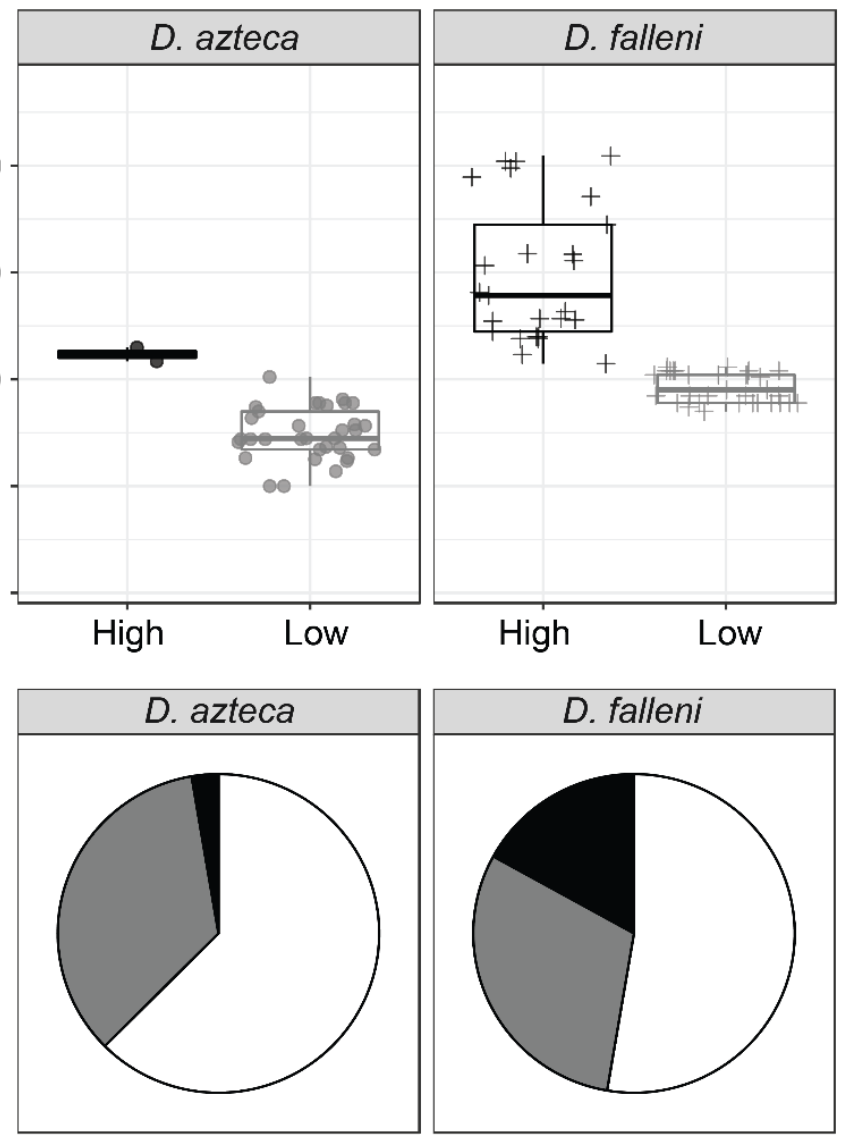

High
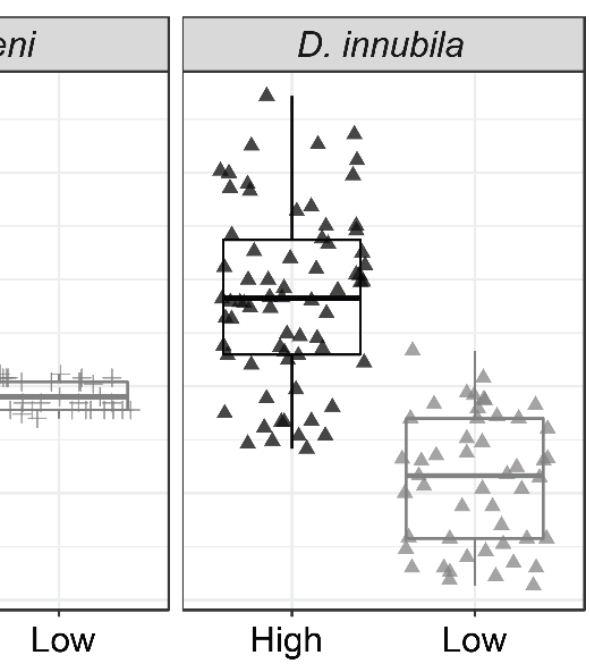

High

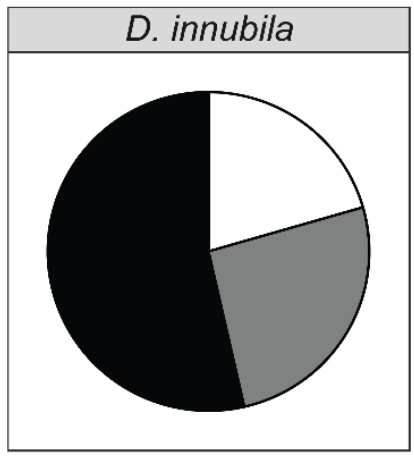

High

Two DiNV types may be maintained due to a trade-off between transmission and virulence

Given the ease that the High type appears to evolve recurrently in populations (Figure 5C), its apparent association with increased infection frequency, and its apparent inability to coinfect with the low type, it is surprising that the High type has not outcompeted the Low type. There are several possible explanations for the maintenance of the two types. First, a soft selective sweep may be occurring on the High type, where recurrent mutation followed by a change in environment changes the fitness of the High type that will eventually result in its fixation (HERMISSON AND PENNINGS 2005). Second, both types may be maintained due to a trade-off (ALIZON AND VAN BAALEN 2008). Such a trade-off might even be associated with frequency-dependent selection and cycling frequencies over time. This trade-off could involve different transmission and virulence strategies or might be related to specific adaptation to different hosts.

In a simple model of viral infection dynamics, the success of the virus is measured by its basic reproduction number $\left(\mathrm{R}_{0}\right)$ which is the ratio of the instantaneous transmission rate $(\beta)$ to the virulence of the virus $(\gamma)$. If there is a trade-off between transmission and virulence, we might expect that although the 
High type has a higher instantaneous rate of transmission, it also has higher virulence, killing infected individuals before they can infect other possible hosts. In contrast, those infected with the Low type persist with the infection and can therefore transmit proportionally more virus due to more interactions with susceptible individuals, despite a lower instantaneous transmission rate. To test this, we simulated populations with two viral types using a modified SIR model in deSolve (SOETAERT et al. 2010). We varied transmission and virulence rates and estimated sets of parameters in which types are maintained within populations. We find that a stable infection frequency depends on both the magnitude of instantaneous transmission rate, and the $\mathrm{R}_{0}$, with higher transmission rates increasing the infection frequency, to a maximum of $1-(\gamma / \beta)$ (Figure 7A). The total infected proportion effectively saturates due to the increased death rate (virulence) of infected individuals, suggesting a trade-off between transmission and virulence as titer increases (Figure 7A). This is consistent with our results in experimental infections in DiNV (Supplementary Figures $7 \& 8$, Cox Hazard Ratio z-value $>2.227, p$-value $<0.02592$ ), and other theoretical treatments (MAY AND NOWAK 1995; ALIZON AND VAN BAALEN 2008). Two types are only maintained when the $R_{0}$ is equal for each type $\left(\gamma_{1} / \beta_{1}=\gamma_{2} / \beta_{2}\right)$. As the transmission rate of the High type increases, it infects a larger proportion of the population and outcompetes the Low type (with High and Low types at equal proportions when the transmission rates equal), the High type proportion saturates due to the equally increasing virulence rate which keeps the $\mathrm{R}_{0}$ equal to the Low type (Figure 7A). Given the requirement for an equal $\mathrm{R}_{0}$ for maintenance, the actual proportion of individuals infected with each type depends on the starting infection frequencies of each type and the difference in absolute transmission rate (Figure 7B). Based on the infection frequencies of our sampled populations, we suspect that the High type was able to evolve earlier in the recently bottlenecked PR population (consistent with the High type background being shared with $94 \%$ of PR Low types), or the absolute transmission rate (and virulence rate) may have increased in PR population, which is likely what has occurred in $\mathrm{CH}$ over time (Figure 7B). Together this suggests that differences in population infection frequencies may depend on a combination of demographic factors, host genetic factors and the instantaneous transmission rate in each population (with lower 411 transmission rates in $\mathrm{HU}$ and SR compared to PR and $\mathrm{CH}$ ). This also implies there is a limit to how virulent 412 a strain can become before it becomes detrimental, as even with higher transmission rates per individual, 413 DiNV may kill the host before it can transmit, reducing its basic reproduction number $\left(\mathrm{R}_{0}\right)$.

\section{Discussion}

415 Viruses are constantly evolving not just to better infect their host, but also to optimize their infection, to 416 infect as many individuals as possible without preventing the transmission to new hosts (MAY AND NOWAK 417 1995; LIPSITCH et al. 1996; ALIZON AND VAN BAALEN 2008). Since the host is also evolving in response 418 to the virus, an evolutionary arms-race often ensues (DAWKINS AND KREBS 1979; KALTZ AND SHYKOFF 
1998; DAUGHERTY AND MALIK 2012). Here, to work towards expanding our understanding of the coevolution of viruses and their hosts, we examine the population dynamics of Drosophila innubila Nudivirus (DiNV), a DNA virus infecting D. innubila (UNCKLESS 2011). DNA viruses have large genomes and often recombination, placing them as a somewhat transitionary pathogen between RNA viruses, bacteria and eukaryotic pathogens and parasites. Within our set of viral samples, we find two DiNV haplotypes which differ by 11 SNPs (Figure 1, named High and Low types). One haplotype (the High type) is associated with higher viral titer, likely due to an increased manipulation of the host immune system and increased expression of viral factors. This derived (High type) has likely recurrently evolved in each population since the last glacial maximum ( $\sim 10,000$ years ago). The two types appear to be incompatible to some degree, as we find little evidence of co-infections, and mutations appear in a similar order as if navigating an epistatic fitness landscape (DOBZHANSKY 1937; KONDRASHOV et al. 2002; GAVRILETS 2004). Finally, despite the higher titer and transmission rate of the High type, we find that the two types are maintained in all populations sampled, possibly because the increased viral titer also increases the virulence, leading to similar basic reproduction numbers in the High and Low type (ALIZON AND VAN BAALEN 2008).

If the reproduction rate, $\mathrm{R}_{0}$, was equal between the two viral types we would expect the infection frequencies to be equal in our sampled populations, which is not the case. This could be caused by the starting frequencies of each type not being equal (with the Low type starting at a higher frequency, Figure 7). There could also be time or host dependent variation, so over time (and between locations), changes in the environment could alter transmission rates of each type, also altering the ratio of High type to Low type.

438 We also do not consider frequency dependent selection in our model, where the transmission rate depends on an interaction between the infection frequencies of the two types.

The $\mathrm{R}_{0}$ could be similar between types, but not identical, resulting in slow changes in the ratio of types over time (as seen between 2001 and 2017, Figures 1D \& 7). As we only have two time points, we

442 could be witnessing a selective sweep of the High type spreading to fixation (NIELSEN 2005), with

443 recombination causing the observed differences in the background. As we find the High type appears to

444 have evolved recurrently, it would be unlikely that we have caught these sweeps partway through in all four 445 populations sampled (Figure 1D). Using the frequency of the High type between 2001 and $2017 \mathrm{CH}$ 446 samples, we can calculate the selection coefficient for the High type if increasing at an exponential rate 447 (which is likely given the intermediate frequency at both time points, Figure 7B). If we assume 5 448 generations per year and an increase among infected individuals from $39 \%$ on 2001 to $71 \%$ in 2017 , the 449 selection coefficient $=0.007$, which suggests the High type would take $\sim 275$ years to fix in a population 450 once it has arisen. Given the coalescence time of the two types is close to the expansion time of the two 451 viruses (2-30 thousand years), this does not fit with our results, suggesting the two types are being 
maintained and not sweeping. Further, if the High type was sweeping, it would be remarkable for us to catch these sweeps occurring in all four populations sampled, given our estimated time to fixation.

Figure 7: Simulated infected populations to determine parameters of stable infection frequencies of two viral types. A. The frequency of stable infection, given the ratio of virulence to transmission, for different magnitudes of transmission but the same starting frequency $(0.001 \%)$. B. Stable infection frequencies given the difference in transmission rates $(\beta)$ between two viral strains, as strain 2 transmission rate (starting

459 frequency $0.001 \%$ ) increases relative to strain 1 transmission rate (starting frequency $25 \%$ ). The difference 460 in infection frequencies of each strain given differing transmission rates of both strains. Stacked bars show 461 the observed frequencies of High type infection (Strain 2, Blue), Low type infection (Strain 1, Red) and 462 Uninfected (Grey) for four sampled populations, positioned on the X-axis to show the estimated ratio of 463 transmission rates for High and Low type, based on the infection frequencies.

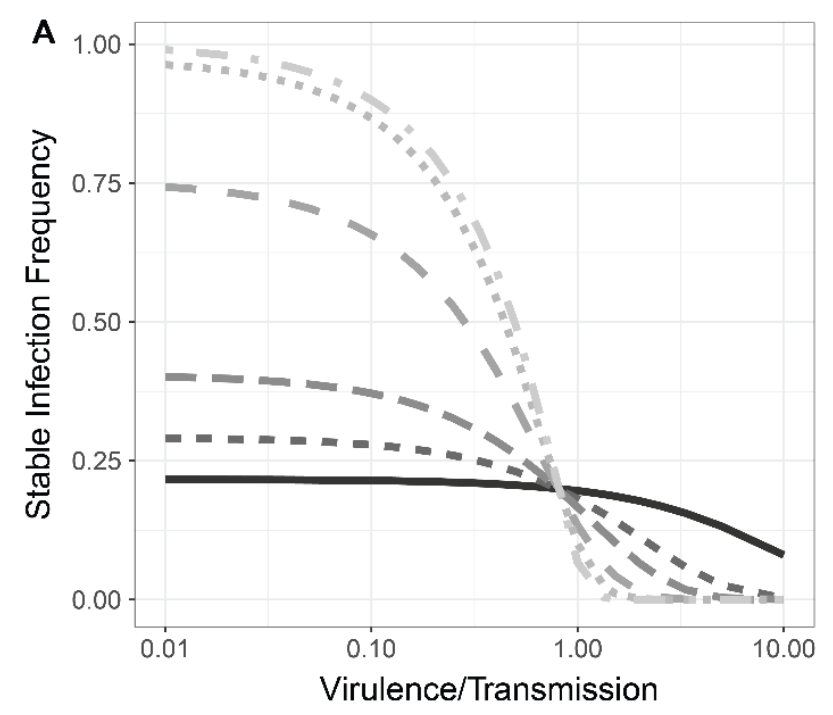

Transmission Rate $=0.0001=0.001=0$
$=0.0005=0.0025=-0.01$

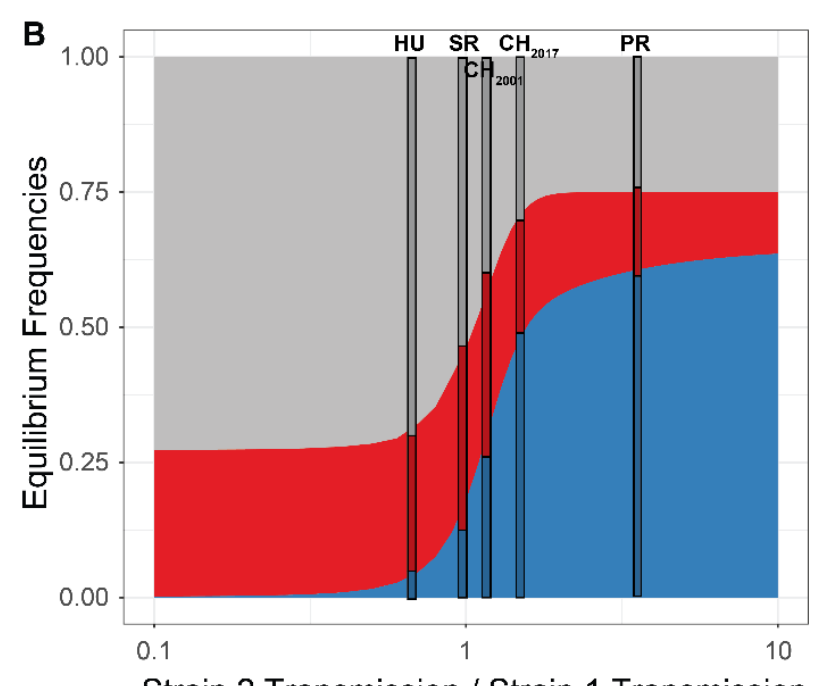

Strain 2 Transmission / Strain 1 Transmission Infection State $\square$ Uninfected $\underset{\text { Infection }}{\text { Strain } 1} \quad \underset{\text { Infection }}{\text { Strain } 2}$

Previous surveys of nudivirus evolution found that a few replication-related genes (including $V L F$ -

468 UNCKLESS 2017; HILL AND UNCKLESS 2018). Given that these repeatedly rapidly evolving genes are also associated with the High and Low types and show the highest levels of adaptation (Figure 1, Figure 4), it is entirely possible that these genes are key factors for infection in nudiviruses. They may also be associated with virulence in other nudiviruses or baculoviruses. It is interesting that we find the same SNPs recurrently evolving in each population, opposed to different SNPs affecting the same genes in each population. 

1990; ROHRMANN 2013), and we find variation in Helicase-2 is associated with viral titer in our survey (Figure 3A \& C). As in other nudiviruses, there is extensive adaptive evolution in the Helicase gene (Figure 4A) (HILl AND UNCKLESS 2017; HILl AND UNCKLESS 2018) which has previously been posited to be associated with change in host range (MAEDA et al. 1993; CROIZIER et al. 1994; ARGAUD et al. 1998; AFONSO et al. 2001). It is possible the Helicase-2 variants allow for optimized infection of different species (CROIZIER et al. 1994). In fact, DiNV infects several Drosophila species in the New World at varying frequencies (including high prevalence in D. munda) (UNCKLESS 2011), and so could have an alternate variant reservoir in any species, with some migration into D. innubila (but not D. azteca or D. falleni), resulting in the appearance of two competing strains. However, this hypothesis ignores the existence of the high and low types which appear to explain the difference in viral titer across multiple species much more convincingly than the Helicase-2 polymorphism (Supplementary Figure 2).

Nudiviruses have extremely high rates of recombination, as they require at least one crossover during their replication (ROHRMANN 2013). Given this high rate of recombination, it is interesting we don't find more intermediate strains with a mix of High and Low SNPs, supporting the idea of an incompatibility or negative epistatic interactions between SNPs of the two types, similar to a Dobzhansky-Muller incompatibility (GAVRILETS 2003).

Some models suggest that viruses are under constant selection to maintain an optimum ratio of virulence to transmission (MAY AND NOWAK 1995; LIPSITCH et al. 1996; ALIZON AND VAN BAALEN 2008). This delicate balance of transmission to virulence could be disrupted with the evolution of a second, hightiter, viral type. In the case of DiNV, the High type has radically higher virulence and appears to compete with the first type impacting the persistence of the virus in a host population. Additionally, the High type appears to recurrently evolve, frequently affecting the persistence of the virus (Figure $5 \& 6$, Supplementary Figure 9). This posits a situation where the optimum strategy for the ancestral viral type is a reduced transmission rate and the fixation of mutations that are incompatible with High type mutations. Further, this fits with the observed consistent order of fixation for mutations that form the High type (Figure 5B), similar to navigating the neutral adaptive landscape between two incompatible forms in a Bateson-DobzhanskyMuller incompatibilities (DOBZHANSKY 1937; ORR 1995; ORR AND TURELLI 2001; GAVRILETS 2003; ORR 2004). This type of viral type interaction is rarely considered in models used for studying infection and could lead to a better understanding of viral dynamics and host-virus co-evolution (JACKSON et al. 2005; JACKSON 2009).

DNA viruses such as DiNV have complicated replication cycles and large genomes. This makes them a sort of evolutionary intermediate between RNA-viruses (small genomes, high mutation rates) and eukaryotes (large genomes, low mutation rates) and tangential to bacteria and archaea (intermediate 
genomes, low recombination rates). However, adaptation appears to occur through changes in a few key proteins. Here we find the evolution of two competing viral types that differ in these few key genes. These viral types are maintained in populations likely due to a trade-off between transmission and virulence. Overall our results suggest that the high mutation rates and extremely high levels of selection can result in the repeated and convergent evolution of novel host-virus interactions. Additionally, we find that these

512 host-virus interactions for large DNA viruses can be much more complicated than previous models suggest 513 (DOLAN et al. 2018; FEDER et al. 2019).

\section{Materials and Methods}

515 Fly collection, DNA isolation and sequencing

516 In this study we used previously collected and sequenced D. innubila (HILL AND UNCKLESS 2020). Briefly

517 we collected these flies across the four mountainous locations in Arizona between the 22nd of August and 518 the 11th of September 2017. Specifically, we collected at the Southwest research station in the Chiricahua 519 mountains ( 5,400 feet elevation, 31.871 latitude -109.237 longitude), Prescott National Forest ( 7,900 520 feet elevation, 34.540 latitude -112.469 longitude), Madera Canyon in the Santa Rita mountains $(\sim 4,900$ 521 feet elevation, 31.729 latitude -110.881 longitude) and Miller Peak in the Huachuca mountains ( 5,900 feet 522 elevation, 31.632 latitude -110.340 longitude). Baits consisted of store-bought white button mushrooms (Agaricus bisporus) placed in large piles about $30 \mathrm{~cm}$ in diameter, at least 5 baits per location. A sweep net was used to collected flies over the baits in either the early morning or late afternoon between one and three days after the bait was set. Flies were sorted by sex and species at the University of Arizona and were flash frozen at $-80^{\circ} \mathrm{C}$ before being shipped on dry ice to the University of Kansas in Lawrence, KS. During these collections we also obtained D. azteca during collections which we also sorted by species and sex and flash froze. D. falleni were collected using a similar method in the Smoky Mountains ( $\sim 6,600$ feet elevation) in shipped on dry ice to the University of Kansas in Lawrence, KS.

For collected D. falleni and D. azteca, we attempted to assess the frequency of DiNV infection 532 using PCR, looking for amplification of the viral gene $p 47$. Using primers from (UNCKLESS 2011), P47F: 533 5'-TGAAACCAGAATGACATATATAACGC and P47R: 5'-TCGGTTTCTCAATTAACTTGATAGC. 534 We used the following conditions: $95^{\circ} \mathrm{C} 30$ seconds, $55^{\circ} \mathrm{C} 30$ seconds, $72^{\circ} \mathrm{C} 60$ seconds per cycle for 35 535 cycles.

536 We sorted 343 D. innubila flies, 60 DiNV positive $D$. falleni and 40 DiNV positive $D$. azteca which 537 we then homogenized and used to extract DNA using the Qiagen Gentra Puregene Tissue kit (USA Qiagen 538 Inc., Germantown, MD, USA). We prepared a genomic DNA library of these 343 DNA samples using a 539 modified version of the Nextera DNA library prep kit ( 350bp insert size, Illumina Inc., San Diego, CA, 
USA) meant to conserve reagents. We sequenced the $D$. innubila libraries on two lanes of an Illumina HiSeq 4000 run (150bp paired-end) (Data to be deposited in the SRA). We sequenced the D. falleni and $D$. azteca libraries on a separate run of a lane of an Illumina HiSeq 4000 (150bp paired-end).

For 80 male Drosophila innubila collected in 2018 (indicated in Supplementary Table 2), we split

544 the sample homogenate in half, isolated DNA from half as described above and isolating RNA using the

545 Direct-zol RNA Microprep protocol (R2061, ZymoResearch, Irvine, CA, USA). We then prepared a cDNA

546 library for each of these 80 RNA samples using a modified version of the Nextera TruSeq library prep kit

547 meant to conserve reagents and sequenced these samples on a NovaSeq NS6K SP 100SE (100bp single

548 end). We also sequenced DNA for these samples, with DNA isolated and prepared as above, also sequenced 549 on a NovaSeq NS6K SP 100SE (100bp single end) (Data to be deposited in the SRA).

\section{$550 \quad$ Sample filtering, mapping and alignment}

551 Following sequencing, we removed primer and adapter sequences using cutadapt (MARTIN 2011) and

552 Scythe (BuFFALO 2018) and trimmed all data using Sickle (-t sanger -q 20 -1 50) (JOSHI AND FASS 2011).

553 We masked the D. innubila reference genome (HILL et al. 2019), using D. innubila TE sequences and

554 RepeatMasker (SMIT AND HuBLEY 2008; SMIT AND HuBLEY 2013-2015). We then mapped short reads to

555 the masked genome and the Drosophila innubila Nudivirus genome (DiNV) (HILL AND UNCKLESS 2018)

556 using BWA MEM (LI AND DURBIN 2009) and sorted using SAMtools (Li et al. 2009). Following this we

557 added read groups, marked and removed sequencing and optical duplicates, and realigned around indels

558 in each mapped BAM file using GATK and Picard (HTTP://BROADINSTITUTE.GITHUB.IO/PICARD ;

559 MCKenNA et al. 2010; DePristo et al. 2011). We considered lines to be infected with DiNV if at least

$56095 \%$ of the viral genome is covered to at least 10 -fold coverage. We then filtered for low coverage and

561 mis-identified species by removing individuals with low coverage of the $D$. innubila genome (less than 5-

562 fold coverage for $80 \%$ of the non-repetitive genome), and individuals we suspected of being misidentified

563 as D. innubila following collection. This left us with 318 D. innubila wild flies with at least 5-fold

564 coverage across at least $80 \%$ of the euchromatic genome, of which 254 are infected with DiNV

565 (Supplementary Table 1). We also checked for read pairs which were split mapped between the DiNV

566 genome and the D. innubila genome using SAMtools.

$567 \quad$ For $D$. falleni we used a previously generated $D$. innubila genome with $D$. falleni variants

568 inserted (HILL et al. 2019). We masked the genome with Repeatmasker (SMIT AND HUBLEY 2013-2015)

569 and mapped short reads to the masked genome, the repeat sequences and the DiNV genome using BWA

570 MEM and SAMtools (LI AND DURBIN 2009; Li et al. 2009). Then, as with D. innubila we filtered for low

571 coverage and mis-identified species by removing individuals with low coverage (less than 5-fold 
coverage for $80 \%$ of the non-repetitive genome) leaving us with 56 D. falleni samples infected with DiNV.

For D. azteca, we downloaded the genome from NCBI (Accession: GCA_005876895.1) which we then called repeats from with RepeatModeler (SMIT AND HUBLEY 2008). We masked the genome with

576 Repeatmasker (SMIT AND HUBLEY 2013-2015) and mapped short reads to the masked genome, the repeat 577 sequences and the DiNV genome using BWA MEM and SAMtools (LI AND DURBIN 2009; LI et al.

578 2009). As with D. innubila we then filtered for low coverage and mis-identified species by removing 579 individuals with low coverage of the D. azteca genome (less than 5 -fold coverage for $80 \%$ of the non580 repetitive genome), which left us with 37 D. azteca samples infected with DiNV. We then called DiNV 581 variation using LoFreq (WILM et al. 2012).

\section{Calling nucleotide polymorphisms across the population samples}

583 For the 318 sequenced samples with reasonable coverage, for host polymorphism, we used the previously 584 generated multiple strain VCF file, generated using a standard GATK HaplotypeCaller/BCFTools pipeline. 585 We used LoFreq (WILM et al. 2012) to call polymorphic viral SNPs within each of the 254 DiNV infected samples, following filtering using BCFtools to remove sites below a quality of 950 and a frequency less than $5 \%$. We then merged each VCF to create a multiple strain VCF file, containing 5,283 SNPs in the DiNV genome. The LoFreq VCF (WILM et al. 2012) output contains estimates of the frequency of each SNP in DiNV in each sample, to confirm these frequencies, in SAMtools (LI et al. 2009) we generated mPileups for each sample and for SNPs of interest (related to viral titer), we counted the number of each nucleotide to confirm the estimated frequencies of these nucleotides at each position in each sample. To

592 confirm that there are no coinfections of types, we also subsampled samples and randomly merged low and 593 high type samples and again generated mPileup files, for SNPs of interest we again counted the number of

594 each nucleotide at each position and confirmed these matched our expected counts in the merged files. We 595 then compared these artificial coinfections to actual samples to confirm the presence or absence of 596 coinfections, finding no samples consistent with coinfections. We then used SNPeff to identify the 597 annotation of each SNP and label synonymous and non-synonymous (CINGOLANI et al. 2012). We extracted 598 the synonymous site frequency spectrum to estimate the effective population size backwards in time using 599 StairwayPlot (LIU AND FU 2015).

600 Identifying differentially expressed genes between DiNV infected and uninfected Drosophila innubila 601 For 100 male Drosophila innubila collected in 2018 (indicated in Supplementary Table 2), we

602 homogenized each fly separately in $100 \mu \mathrm{L}$ of PBS. We then split the sample homogenate in half, isolated 603 DNA from half as described above and isolating RNA using the Direct-zol RNA Microprep protocol 604 (R2061). Using the isolated DNA, we tested each sample for DiNV using PCR for P47 as described 
605 previously, using 40 DiNV infected samples and 40 uninfected samples. We then prepared a cDNA

606 library for each of these 80 RNA samples using a modified version of the Nextera TruSeq library prep kit

607 meant to conserve reagents and sequenced these samples on a NovaSeq NS6K SP 100SE (100bp single

608 end). We also sequenced DNA for these samples, with DNA isolated and prepared as above, also

609 sequenced on a NovaSeq NS6K SP 100SE (100bp single end). The DNA sequenced here was mapped as

610 described above, with variation called as described above for other DNA samples.

611 Following trimming and filtering the data as described in the methods, we mapped all mRNA

612 sequencing data to a database of rRNA (QUAST et al. 2013) to remove rRNA contaminants. Then we

613 mapped the short read data to the masked $D$. innubila genome and DiNV genome using GSNAP (-N 1 -o

614 sam) (WU AND NACU 2010). We estimated counts of reads uniquely mapped to D. innubila or DiNV

615 genes using HTSEQ (ANDERS et al. 2015) for each sample. Using EdgeR (ROBINSON et al. 2009) we

616 calculated the counts per million (CPM) of each gene in each sample and counted the number of samples

617 with CPM $>1$ for each gene. We find that over $70.3 \%$ of genes have a CPM $>1$ in at least 70 samples.

618 For the remaining genes, we find these genes are expressed in all samples of a subset of the strains (e.g.

619 DiNV uninfected, DiNV infected, DiNV high infected, DiNV low infected). This supports the validity of

620 the annotation of D. innubila, given most genes are expressed in some manner, and suggests our RNA

621 sequencing samples show expression results consistent with the original annotation of the D. innubila

622 genome.

623 We attempted to improve the annotation of the D. innubila genome to find genes expressed only

624 under infection. We extracted reads that mapped to unannotated portions of the genome and combined these

625 for uninfected samples, samples infected with high type DiNV and samples infected with low type DiNV

626 as three separate samples. We then generated a de novo assembly for each of these three groups using

627 Trinity and Velvet (SCHULZ et al. 2012; HAAS et al. 2013). We then remapped these assemblies to the

628 genome to identify other transcripts and found the consensus of these two for each sample. Using the

629 Cufflinks pipeline (GHOSH AND CHAN 2016), we mapped reads to the D. innubila genome and counted the

630 number of reads mapping to each of these putative novel transcript regions, identifying 15,676 regions of

631 at least $100 \mathrm{bp}$, with at least 1 read mapping in at least 1 sample. Of these, 717 putative genic regions have

632 at least 1 CPM in all 80 samples, or in all samples of one group (DiNV uninfected, DiNV infected, DiNV-

633 low infected, DiNV-high infected). We next attempted to identify if any of these genes are differentially

634 expressed between types, specifically between uninfected strains and DiNV infected strains, and between

635 low-type infected and high-type infected strains. Using a matrix of CPM for each putative transcript region

636 in each sample, we calculated the extent of differential expression between each type using EdgeR

637 (ROBINSON et al. 2009), after removing regions that are under expressed, normalizing data and estimating

638 the dispersion of expression. We find that 26 putative genes are differentially expressed between infected 
and uninfected types, and 69 putative genes are differentially expressed between high and low types. We took these regions and identified any homology to D. virilis transcripts using blastn (ALTSCHUL et al. 1990). We find annotations for 37 putative genes are either expressed in all samples, or differentially expressed between samples. Of the 14 putative genes expressed in all samples, nine have the closest blast hit to an rRNA gene, and five have hits to unknown genes. For 23 differentially expressed putative genes with blast hits, 3 genes are like antimicrobial peptides (IM1,IM14,IM3), these genes are significantly downregulated upon infection, like other Toll regulated AMPs, and have significantly lower expression in strains infected with high type DiNV compared to low types. The remaining 20 genes all have similarity to genes associated with cell cycle regulation, actin regulation and tumor suppression genes.

\section{Identifying genes associated with viral titer in Drosophila innubila}

As the logarithm of viral titer was normally distributed (Shapiro-Wilk test $\mathrm{W}=0.05413, p$-value $=0.342$ ), we used PLINK (PURCELL et al. 2007) to associate nucleotide polymorphism to logarithm of viral titer in infected samples. We fit a linear model in PLINK including population, sex, Wolbachia presence, the date of collection and the relationship matrix for relationship of each sample (inferred using PLINK).

We first fit this model for all 5,283 viral polymorphisms, before performing the association study, we also pruned viral SNPs for both the total population and each subpopulation leaving 1,403 SNPs. For the total sample we identified associations between the logarithm of viral titer and the frequency of the viral polymorphism in each individual sample, resulting in the following model:

$$
\begin{gathered}
\log _{10}(\text { viral titre }) \sim S N P+h s+w+p+d c+(S N P * h s)+(S N P * p)+(S N P * w) \\
+ \text { relationship }[\text { strain }]
\end{gathered}
$$

Where hs $=$ host sex, $\mathrm{p}=$ location of collection, $\mathrm{w}=$ Wolbachia presence, $\mathrm{dc}=$ date collected

Following model fitting, we found factors which seemed to show little or no effect on viral titre ( $p$-value $>$ 0.1) using an ANOVA in R (TEAM 2013), and removed these, refitting the model. This was done step-wise, leaving the following model by the end:

$$
\log _{10}(\text { viral titre }) \sim S N P+h s+p+(S N P * h s)+\text { relationship [strain] }
$$

Following this we also performed a GWAS using PLINK (PURCELL et al. 2007) in the host, using previously called host variation, and considering viral haplotype as an additional covariate.

$$
\log _{10}(\text { viral titre }) \sim S N P+h s+p+(S N P * h s)+v h+\text { relationship [strain] }
$$

Where hs $=$ host sex, $\mathrm{p}=$ location of collection, $\mathrm{w}=$ Wolbachia presence, $\mathrm{dc}=$ date collected, $\mathrm{vh}=$ viral haplotype. We found no convincing significant associations (Supplementary Figure 11).

We repeated this analysis for DiNV variants in D. azteca and D. falleni separately. We performed the GWAS twice, first using the original model, then including viral haplotype as an additional covariate. 
672 Following the identification of the viral haplotype associated with viral titre we sought to determine the

673 effect of viral haplotypes in actual infections. For 20 samples with fly homogenate, we determine the viral

674 titer and haplotype following filtration with a $0.22 \mu \mathrm{M}$ filter.

675 We performed qPCR for the viral gene $p 47$ (Forward 5-TCGTGCCGCTAAGCATATAG-3, 676 Reverse 5-AAAGCTACATCTGTGCGAGG-3) on $1 \mu \mathrm{L}$ of fly filtrate per sample and compared the 677 estimated $\mathrm{Cq}$ values across 3 replicates to estimated viral copy number to confirm viral concentration 678 (protocol: 2 minutes at $95^{\circ} \mathrm{C}, 40$ cycles of $95^{\circ} \mathrm{C}$ for 30 seconds and $59^{\circ} \mathrm{C}$ for 20 seconds, followed by 2 679 minutes at $72^{\circ} \mathrm{C}$ ). Following this we diluted samples to similar Cq values, relative to the sample with the 680 highest $\mathrm{Cq}$ value. We confirmed this by repeating qPCR with $p 47$ primers of $1 \mu \mathrm{L}$ of each sample.

For each filtrate sample we performed infections on 30 D. innubila males 4-5 days following emergence using pricks with sterile needles dipped in viral filtrate. We recorded survival of each fly each day and removed dead flies. Finally, we took samples 1, 3 and 5-days post infection and measured viral copies of $p 47$ relative to tpi at each time point.

Phylogeography of DiNV infection and the evolution of the different viral haplotypes

For each DiNV infected D. innubila sample, we reconstructed the consensus DiNV genome infecting them using GATK AlternateReferenceMaker and the VCF generated for each strain (MCKENNA et al. 2010; DEPRISTO et al. 2011). We then converted these genomes into Phylip format and used BEAST2 to build the phylogeny of DiNV genomes using 100 million iterations with a burn in of 5 million (BOUCKAERT et al. 2014). We considered phylogeography by providing the longitude and latitude of each samples collection. We then generated a final consensus phylogeny using Tracer with at least 90\% majority consensus and removed trees that had not converged to the same joint density (BOUCKAERT et al. 2014).

693 To reconstruct the evolution of the high type viral type we used APE (PARADIS et al. 2004) to infer the 694 appearance order of the six perfectly linked SNPs on the phylogeny using the all different rates (ARD) 695 discrete model (PARADIS et al. 2004). We also confirmed these recurrent mutations across the phylogeny 696 using TreeTime to identify recurrently evolving SNPs (SAGULENKO et al. 2018). Finally, we also created 697 a matrix of SNPs present in at least 2 viral samples and used this matrix in a principle component analysis 698 in R (TEAM 2013), labelling each sample by their viral type in the PCA. Simulating the evolution of the high and low viral haplotypes.

700 We sought to simulate the infection of DiNV in D. innubila when considering the evolution of a high titer 701 viral haplotype, specifically if two viral types can be maintained against each other at stable frequencies, 702 and if the high viral haplotype with 5 shared mutations could evolve recurrently in the given time period 703 given realistic parameters. We used the R package DeSolve (SOETAERT et al. 2010) to simulate infection 
dynamics in a modified SIR model. Specifically, we removed a resistant class, under the assumption that flies won't live long enough to shed the infection. Therefore, the proportion of population infected per generation is as follows:

$$
p_{\text {Infected }}=(\text { Susceptible } * \text { Infected } * \beta)-(\text { Infected } * \gamma)
$$

708 Where $\beta$ represents an infection parameter and $\gamma$ represents a virulence parameter (e.g. the increased 709 likelihood an infected individual has of dying before it can spread its infection). This equation can be rearranged to show the stabilized maximum frequency of infection:

$$
p_{\text {Infected }}=1-\frac{\gamma}{\beta}
$$

712 Which is maintained while $\gamma$ is greater than 0.01 . The average frequency decreases as absolute transmission

713 rate decreases past this point. We then extended this to include two competing infection types, to represent

714 the two viral types, with the total proportion of population infected per generation as follows:

$$
\begin{aligned}
p_{\text {Susceptible }}=1 & -\left(\text { Susceptible } * \text { Infected }_{1} * \beta_{1}\right)-\left(\text { Infected }_{1} * \gamma_{1}\right) \\
& +\left(\text { Susceptible } * \text { Infected }_{2} * \beta_{2}\right)-\left(\text { Infected }_{2} * \gamma_{2}\right)
\end{aligned}
$$

717 This equation can be rearranged as before to show the stabilized frequency of infection for two viral types:

$$
p_{\text {Infected }}=1-\frac{\gamma_{1}+\gamma_{2}}{\beta_{1}+\beta_{2}}
$$

In this case, the two viral haplotypes can both be maintained within a population when the ratio of infection to virulence are the same:

$$
\frac{\gamma_{1}}{\beta_{1}}=\frac{\gamma_{2}}{\beta_{2}}
$$

722 We assessed how starting frequency and difference in $\gamma$ and $\beta$ affects the evolution of each type and their stable frequencies but repeating these simulations for 10,000 generations for each set of parameters. We repeated simulations with transmission rates varying between 0.00001 and 1 , and virulence rates set as the transmission rate, divided by a scaling factor between 0.01 and 10 (to vary virulence at multiple rates higher and lower than transmission rate), with all pairwise combinations across all parameters.

Next, we attempted to assess if the 'high titer' viral haplotype could possibly evolve recurrently in 728 each population in the time scale seen in our findings. We again used the modified SIR model, this time 729 discrete with population sizes set to 1 million individuals, based on StairwayPlot estimates (LIU AND FU 730 2015). For each infected individual in the population, we tracked the viral titer and also recorded the 731 presence of absence of five mutations, with each mutation increasing the titer of infection, but each further 732 mutation having successively smaller increases in viral titer (titer $\sqrt{\text { no.muts }}$ ), representing the epistatic 733 interaction of high titer associated mutations seen in the viral haplotype. We multiplied the infection 734 parameter, mutation parameter and virulence parameter by viral titer, under the assumption that viral titer 735 increases both infection and death rate, and the mutation rate is per viral particle. We considered a per site 
mutation rate of $10^{-6}$, based on estimated baculovirus mutation rate (ROHRMANN 2013; CHATEIGNER et al. $2015)$, with a specific mutation rate for the five haplotype mutations of $6.4 \mathrm{e}-12(1 \mathrm{e}-6 / 155 \mathrm{kbp}) *$ viral titer. We then simulated populations in replicate 1000 times for 100,000 generations with a starting infection frequency of $10 \%$ for the 'low titer' haplotype, recording the frequency of the virus in a population, the frequency of the haplotype and the time that each 'high titer' mutation reaches high enough frequency to escape stochastic behavior and behave deterministically under selection (GILLESPIE 2004).

To estimate the possible selection coefficient for DiNV in the $\mathrm{CH}$ population, we assumed an exponential distribution and 5 viral generations per year ( 80 generations between 2001 and 2017). We then

744 solved the following equation:

$$
P_{2017}=P_{2001} *(1+s)^{t}
$$

Where $\mathrm{P}_{2017}=$ the frequency of the High type among viral samples in $2017(71 \%), \mathrm{P}_{2001}=$ the frequency of the High type among viral samples in $2001(39 \%), \mathrm{s}=$ the selection coefficient and $\mathrm{t}=$ the number of generations (80). We then used this estimated selection coefficient in the same equation to find the number of generations ( $\mathrm{t}$ ) to go from $1 / 2 \mathrm{~N}_{\mathrm{e}} \mathrm{S}\left(0.0000714\right.$, assuming an $\mathrm{N}_{\mathrm{e}}$ of 1000000$)$ to fixation $(0.99)$ :

$$
0.99=0.0000714 *(1+0.007)^{t}
$$

\section{Experimental infections of Drosophila innubila with DiNV}

752 We chose D. innubila samples infected with DiNV and with sequenced genomes, 4 infected with the high 753 type DiNV and 4 infected with the low type. For these samples we estimated their viral copy number per

754 host genome as described previously. We used qPCR on $p 47$ and tpi to find the differences in Cq values to 755 calculate the concentration of each sample relative to the lowest concentration sample and diluted $50 \mu \mathrm{L}$ of 756 filtrate for each sample to match the concentration of each sample to the samples with the lowest titer 757 (IPR07). For a separate $50 \mu \mathrm{L}$ of the IPR01 sample, we performed 1 in 10 serial dilutions to give $45 \mu \mathrm{L}$ of 758 filtrate at full concentration, 1 in 10 concentration, 1 in 100 concentration and 1 in 1000 concentration. 759 Using these sets of samples (matched titer and serial dilutions) we next performed experimental infections.

We transferred 50 D. innubila (of roughly equal sex ratio) to new food and let them lay eggs for 1 week, following this we collected male offspring aged 2-5 days for experimental infections.

763 flies with each sample. For flies in batches of 10, we performed pricks with microneedles dipped in the 764 prepared viral filtrate. For each day we also had 2 control replicates of 10 flies pricked with microneedles 765 dipped in sterile media. Following infections, we checked on each vial of 10 flies one-hour post infection 766 and removed dead flies (likely killed by the needle instead of the virus). We also checked each vial each 767 morning for 15 days, removing dead flies (freezing to determine the viral titer), and flipping flies to new 
768 food every 3-4 days. Checking at 10am each day, we recorded the day that each fly died, what filtrate they

769 had been infected with, and what replicate/infection day set they belonged to. We next looked for

770 differences in survival over time compared to sterile wound controls using a Fit proportional hazards

771 regression model in R (TEAM 2013; KASSAMBARA et al. 2017), considering titer, infection sample and

772 replicate as co-variates (day of death $\sim$ [titer or strain] + infection date).

773 For a second set of experimental infections (performed as described above, stabbed with diluted

774 filtrate from different strains), we also removed 3 living flies 1 hour, 1 day and 5 days post infection. Using

775 qPCR, we found the difference in $p 47 \log -\mathrm{Cq}$ and tpi log-Cq to estimate the viral copy number for each

776 sample over time.

\section{Acknowledgements}

778 This work was completed with helpful discussion from Justin Blumenstiel, Joanne Chapman, John Kelly,

779 Stuart MacDonald, Andrew Mongue and Carolyn Wessinger. We would especially like to thank Maria

780 Orive, Kelly Dyer and Paul Ginsberg for helpful feedback in the writing of the manuscript and framing of 781 the discussion. Collections were completed with assistance from Todd Schlenke, Paul Ginsberg, Kelly Dyer,

782 Brandon Cooper, John Jaenike and the Southwest Research Station. We thank Brittny Smith and Jenny 783 Hackett at the KU CMADP Genome Sequencing Core (NIH Grant P20 GM103638) and K-INBRE 784 Bioinformatics Core for assistance in genome isolation, library preparation, sequencing and computational 785 resources. This work was supported by a K-INBRE postdoctoral grant to TH (NIH Grant P20 GM103418). 786 This work was also funded by NIH Grants R00 GM114714 and R01 AI139154 to RLU. D. falleni collection 787 was funded by NSF grant DEB-1737824. 


\section{Supplementary Figures}

789 Supplementary Figure 1: McDonald-Kreitman based statistics for each gene in population of Drosophila 790 innubila Nudivirus, with viral envelope and GrBNV potential virulence factors shown separately. DoS = 791 direction of selection, Selection Effect $=$ SnIPRE estimated weighted DoS. Boxplots marked with a * are 792 significantly higher than background/other viral genes (GLM $p$-value $<0.05$ ).

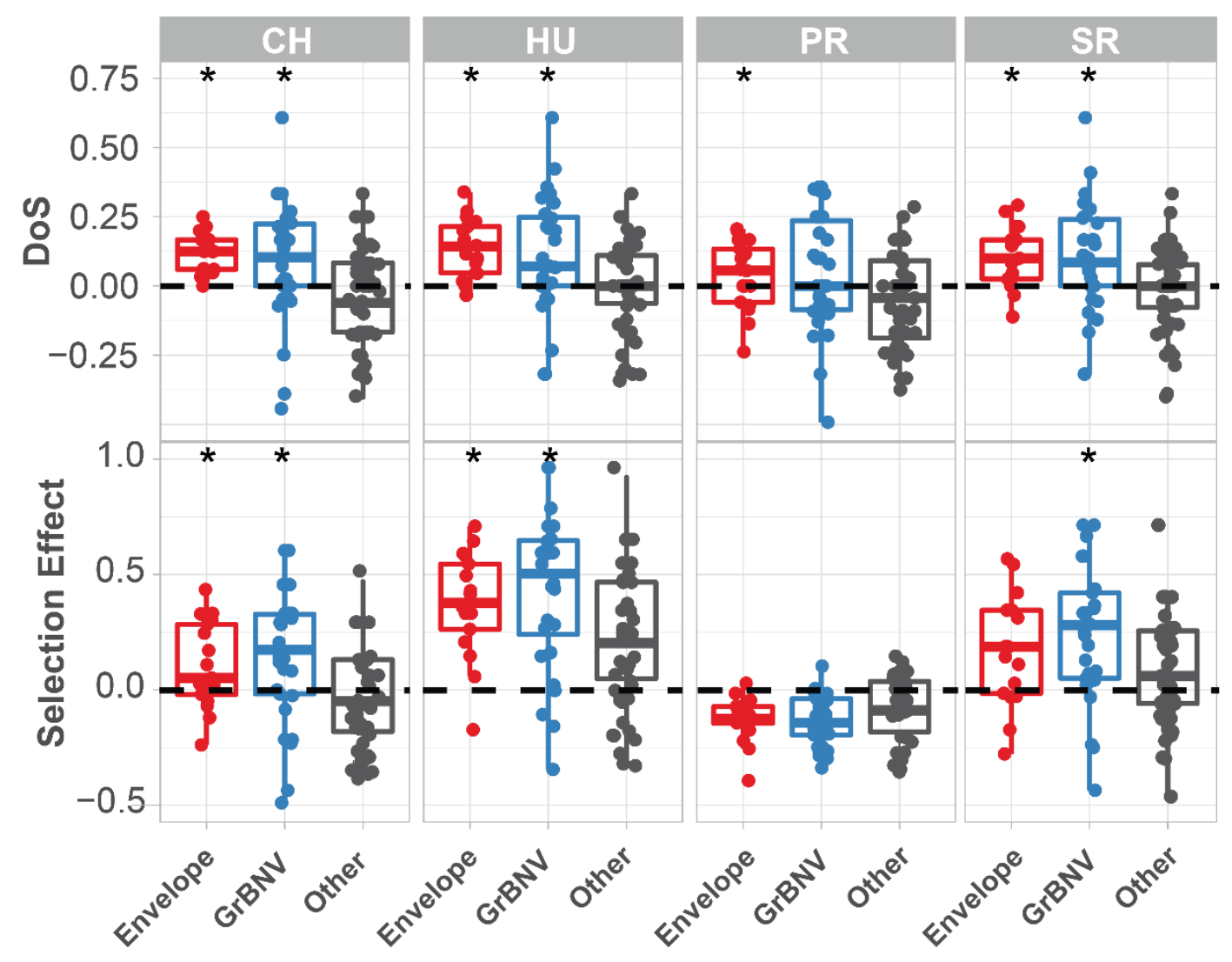


794 Supplementary Figure 2: Frequency of each significant SNP within each sample, ranked by the viral titer 795 in each sample $($ left = lowest, right $=$ highest $)$, to show the strong linkage of SNPs and little evidence of co-

796 infection, also highlights the association between SNP frequency and Helicase-2.

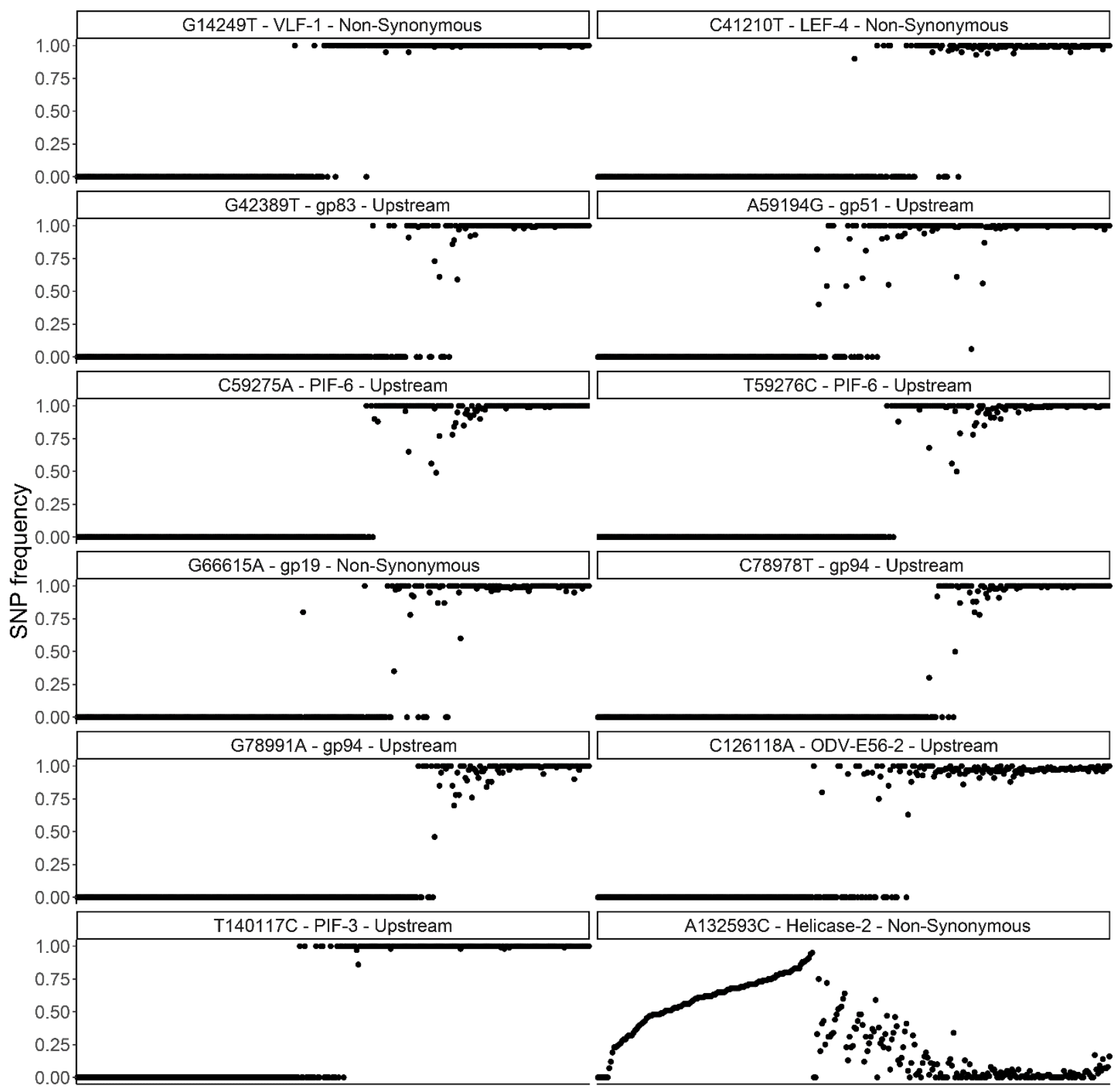


bioRxiv preprint doi: https://doi.org/10.1101/2020.05.14.096024; this version posted May 19, 2020. The copyright holder for this preprint (which was not certified by peer review) is the author/funder, who has granted bioRxiv a license to display the preprint in perpetuity. It is made available under aCC-BY-NC-ND 4.0 International license.

798 Supplementary Figure 3: Linkage disequilibrium between SNPs in DiNV. The labelled SNPs

799 (significant SNPs found in the GWAS) are strongly linked. Points are colored by the estimated linkage

800 between SNPs, from red (highly linked, $r^{2}=1$ ) to white (unlinked, $r^{2}=0$ )

\section{Pairwise LD}

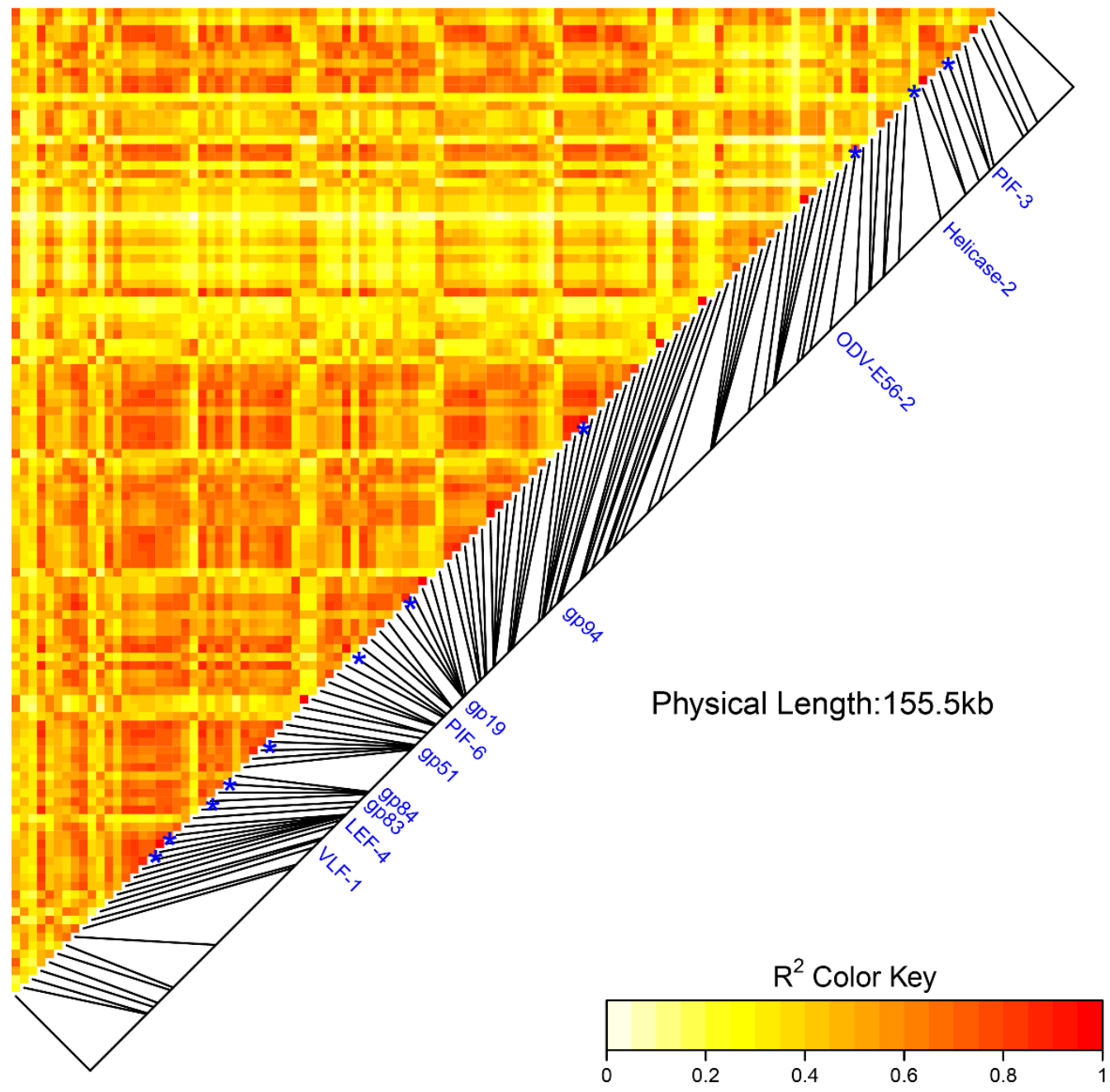


802 Supplementary Figure 4: Linkage $\left(\mathrm{r}^{2}\right)$ between different types of SNPs in each population of DiNV, and

803 across all samples. Other $=$ SNPs which are not significantly associated with DiNV titer and do not form

804 the viral haplotype. Sig = SNPs which are significantly associated with DiNV titer and do not form the

805 viral haplotype. $\mathrm{BG}=\mathrm{SNPs}$ which are in the background which the viral haplotype evolved on in each

806 population.
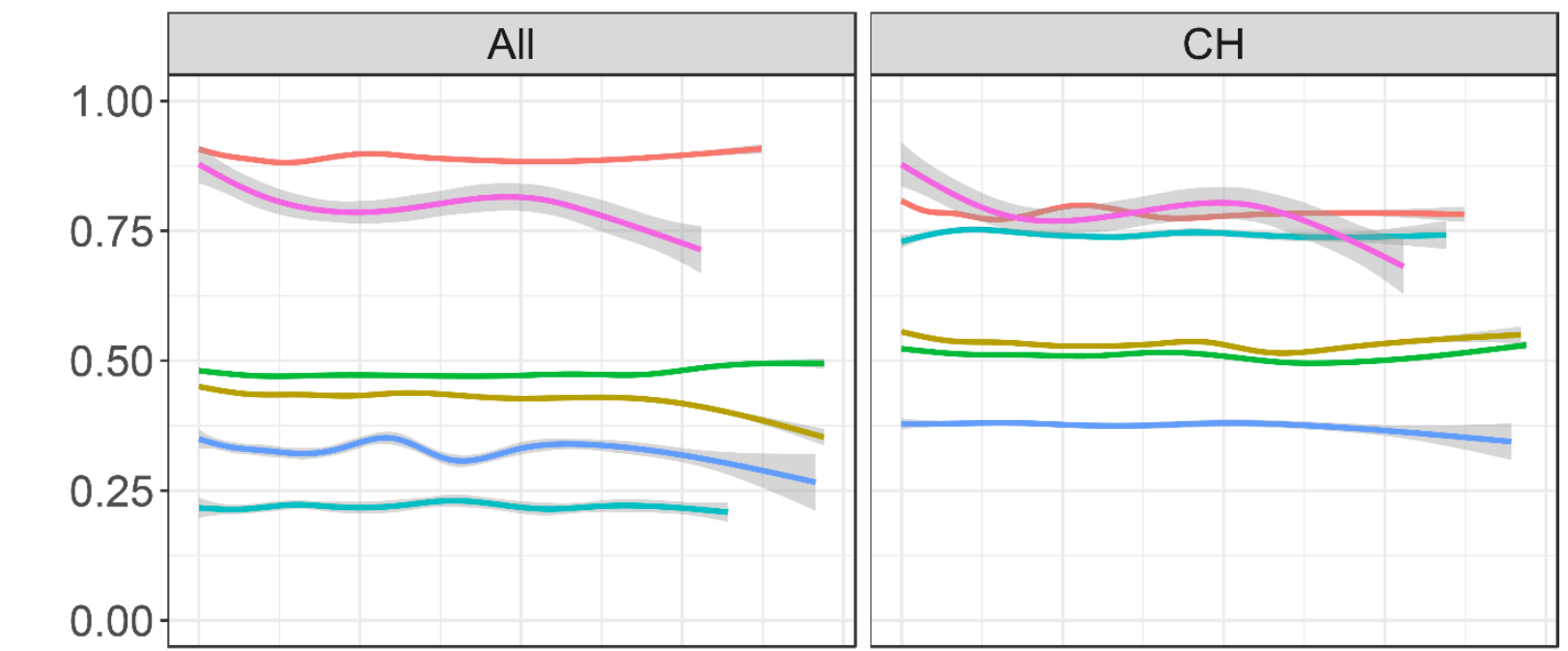

\section{ข}
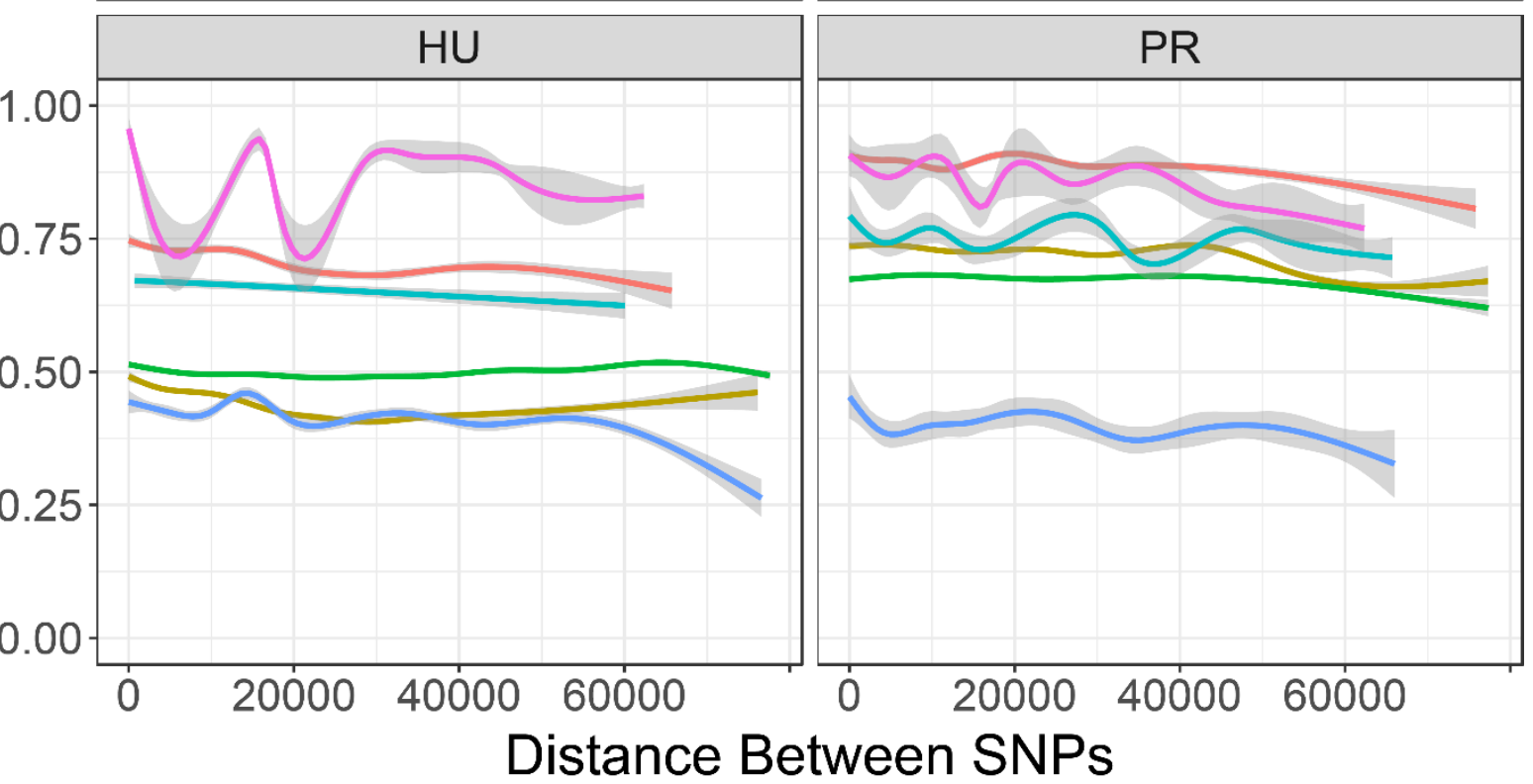
808 Supplementary Figure 5: Volcano plot of changes in gene expression between $D$. innubila infected with

809 DiNV and uninfected controls. Gene categories of interest, such as enriched categories, are highlighted in

810 color. The FDR-correct significance cut-off of 0.01 (10,320 tests) is shown as a dashed line. B. Comparison

811 of gene expression changes upon infection for D. innubila and D. melanogaster. Significantly differentially

812 expressed genes ( $p$-value $<0.01$, FDR-corrected) are colored, genes differentially expressed in both species

813 are colored blue, genes differentially expressed in just D. melanogaster are colored yellow and genes

814 differentially expressed in just $D$. innubila are colored red.
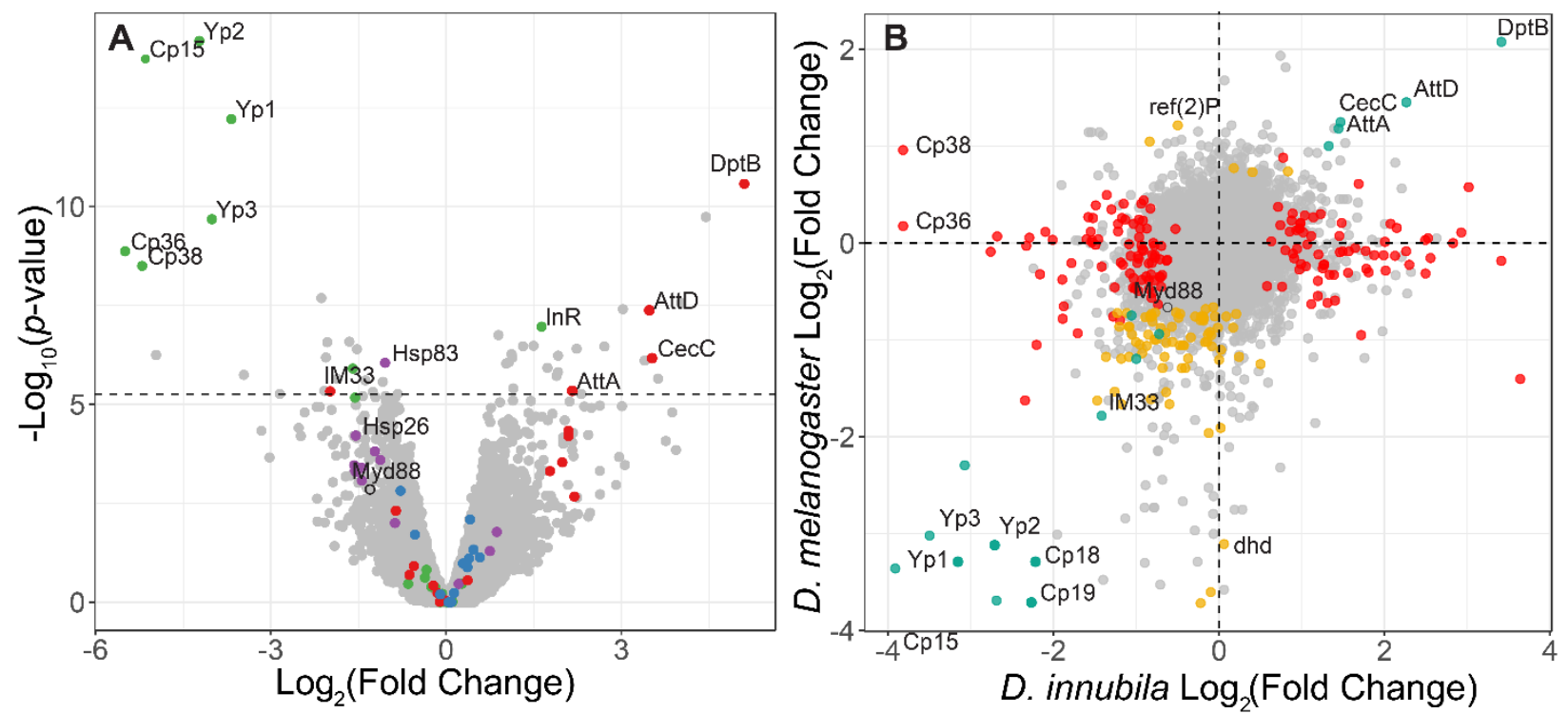
816 Supplementary Figure 6: Expression changes (shown as transcript fragments per 1 million reads per 1kbp

817 of exon) of antimicrobial peptides between strains infected with high type DiNV, low type DiNV or not

818 infected. Black stars above low samples show significant differential expression between DiNV infected

819 strains and uninfected strains (multiple testing corrected $p$-value $<0.05$ ). Red stars above high samples

820 show significant differential expression between low type infected strains and high type infected strains

821 (multiple testing corrected $p$-value $<0.05$ ).
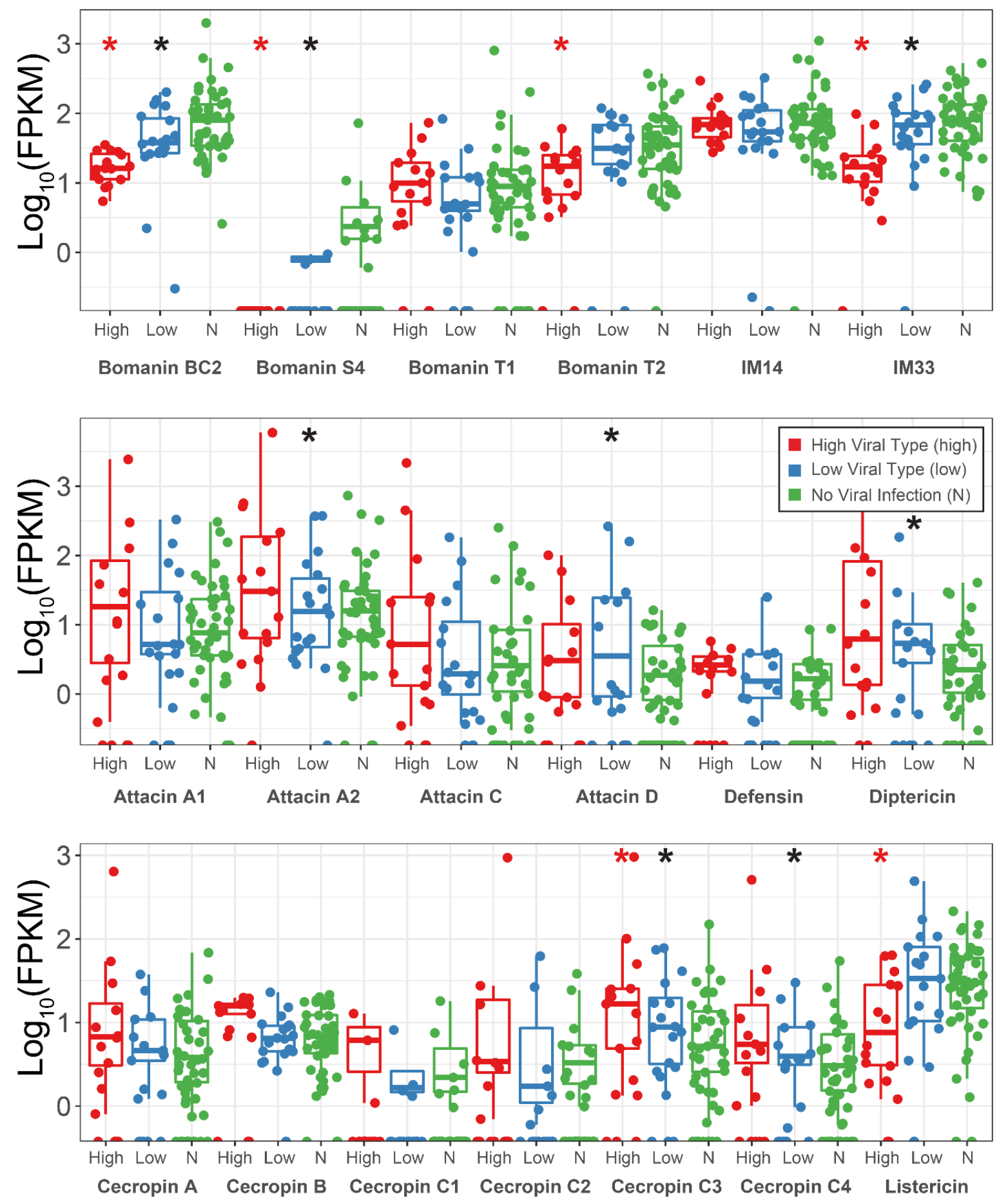
823 Supplementary Figure 7: Effect of differences in viral type and titer in experimental infections. A.

824 Survival curves of D. innubila infected with DiNV filtrate of different dilutions compared to control flies

825 pricked with sterile media, for 15 days post infection. B. qPCR copy number of viral $p 47$ relative to tpi in

826 samples of D. innubila infected with DiNV filtrate of different dilutions, between 1 and 1000 viral particles

827 per host genome copy.

A

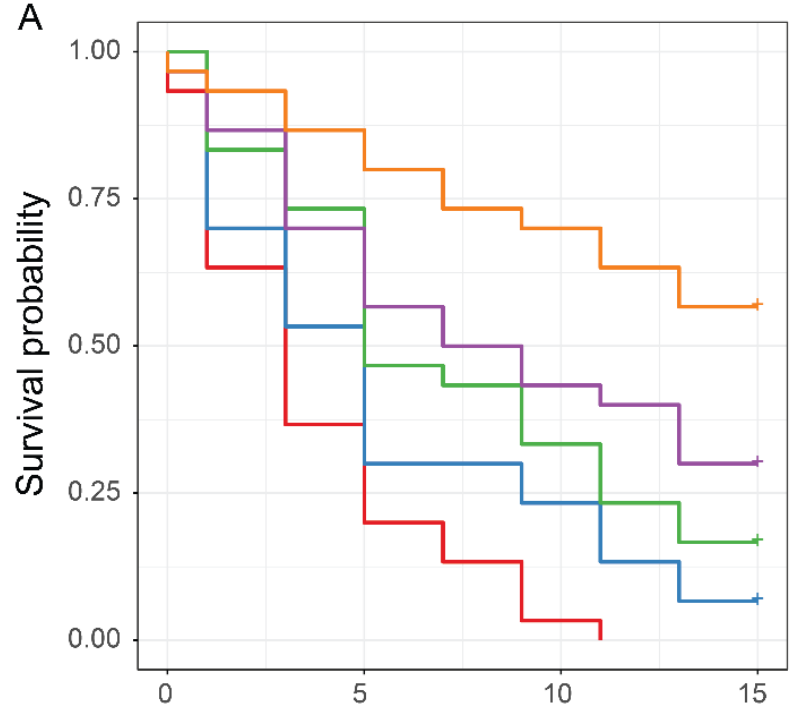

$\mathrm{B}$

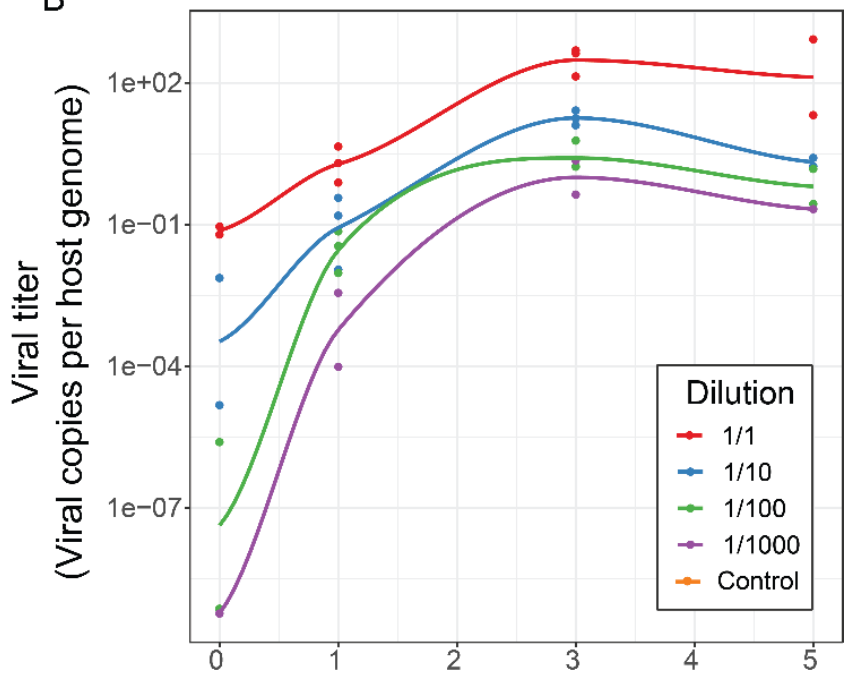

Days Post Infection 
829 Supplementary Figure 8 A: Survival of $D$. innubila reference strain 5 days post infection, using filtrate

830 from different samples versus uninfected control, colored by high type virus or low type virus. B. Survival

831 of $D$. innubila reference strain 5 days post infection using serial dilutions of IPR01 filtrate versus control.

832 C. Viral titer estimated per viral genotype at 5 days post-infection, colored by high type virus or low type

833 virus. D. Viral titer of DiNV infecting D. innubila reference strain 5 days post infection using serial

834 dilutions of IPR01 filtrate versus control.
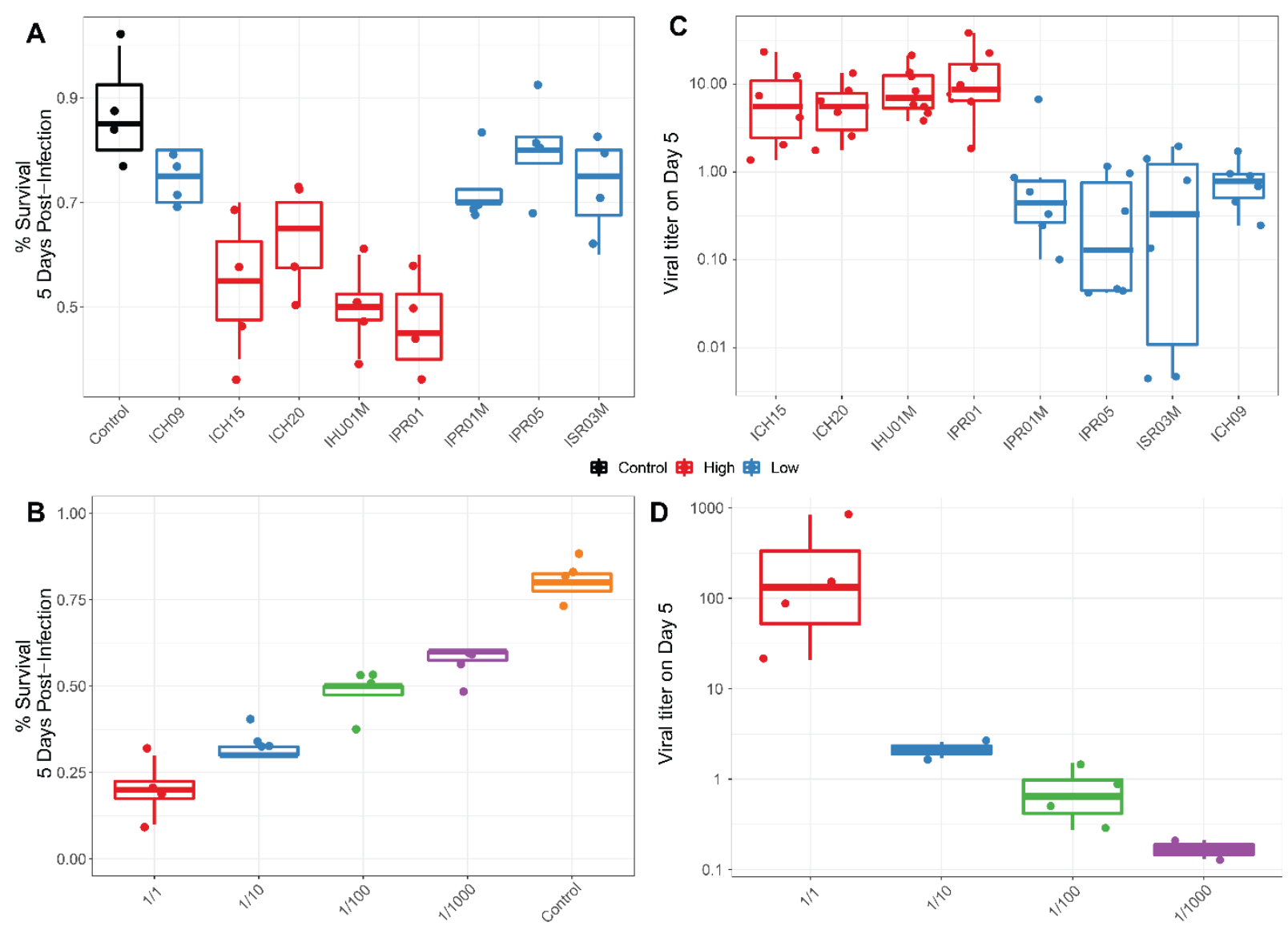
836 Supplementary Figure 9. A. Frequency of samples with different numbers of SNPs in the viral

837 haplotype, there ae very few intermediate types. B. Frequency of each SNP in samples infected with the

838 virus, showing there is little evidence of co-infections. C. Principle component analysis of DiNV strains

839 using variation of strains. Strains are colored by the viral type, showing its recurrent evolution. Point

840 shape denotes species in which DiNV was found (D. azteca, D. falleni or D. innubila). Strains cluster by

841 collection location. D. Linkage between SNPs in the viral haplotype $\left(\mathrm{r}^{2}\right)$ and other SNPs in the haplotype,

842 to other SNPs in the viral genome.
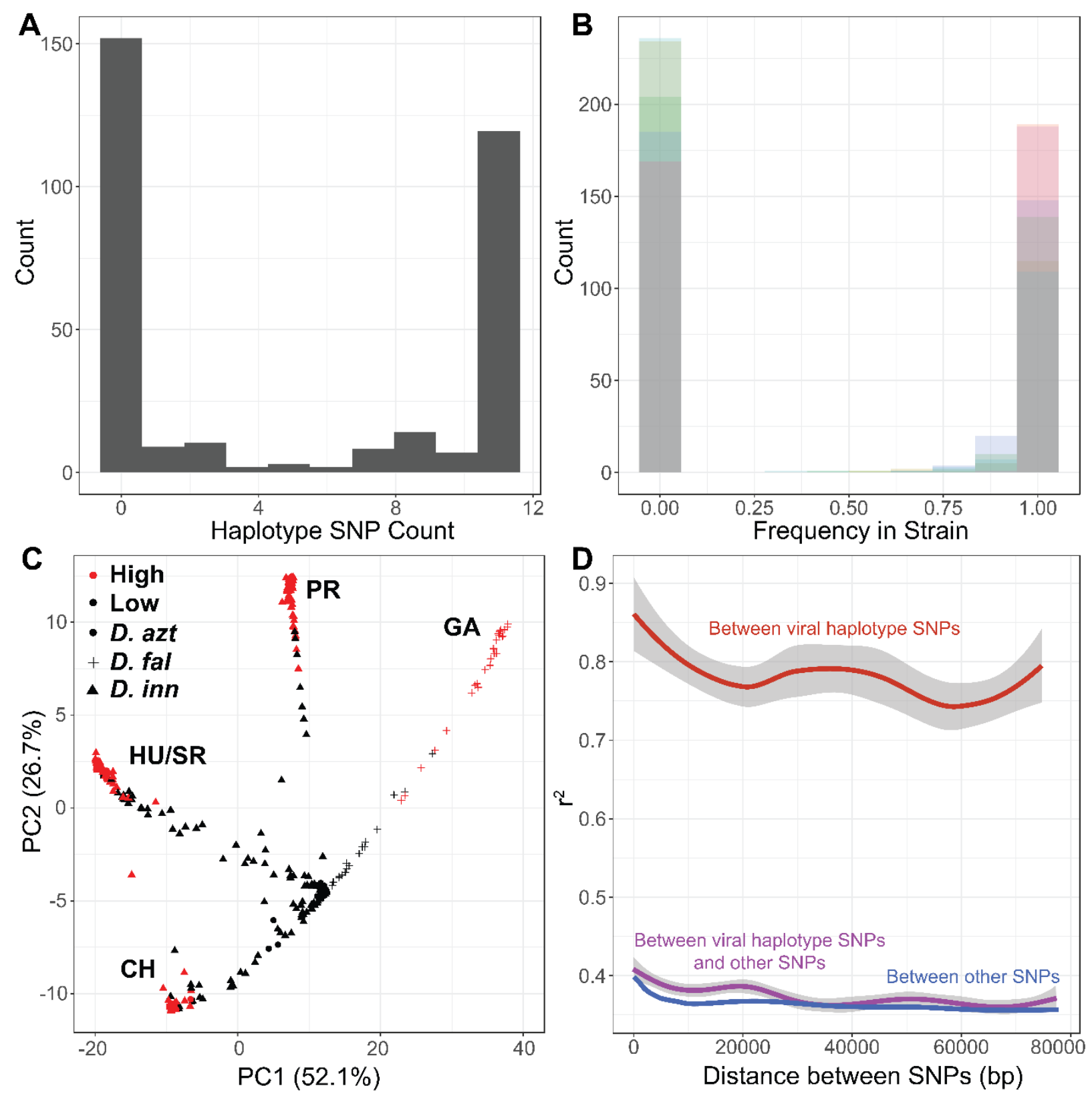
844 Supplementary Figure 10. A: Manhattan plot of significance of SNP on viral titer after factoring in

845 interaction with the viral haplotype. The significance cut offs are labelled ( $p$-value $<0.05$ after multiple

846 testing correction dotted, $p$-value $<0.05$ after permutations dashed). B: Manhattan plot of SNP x viral

847 haplotype interaction for viral titer GWAS in D. innubila, calculated using PLINK. The significance cut

848 offs are labelled ( $p$-value $<0.05$ after multiple testing correction dotted, $p$-value $<0.05$ after permutations

849 dashed). C: Manhattan plot of SNP x sex interaction for viral titer GWAS in D. innubila, calculated using

850 PLINK.

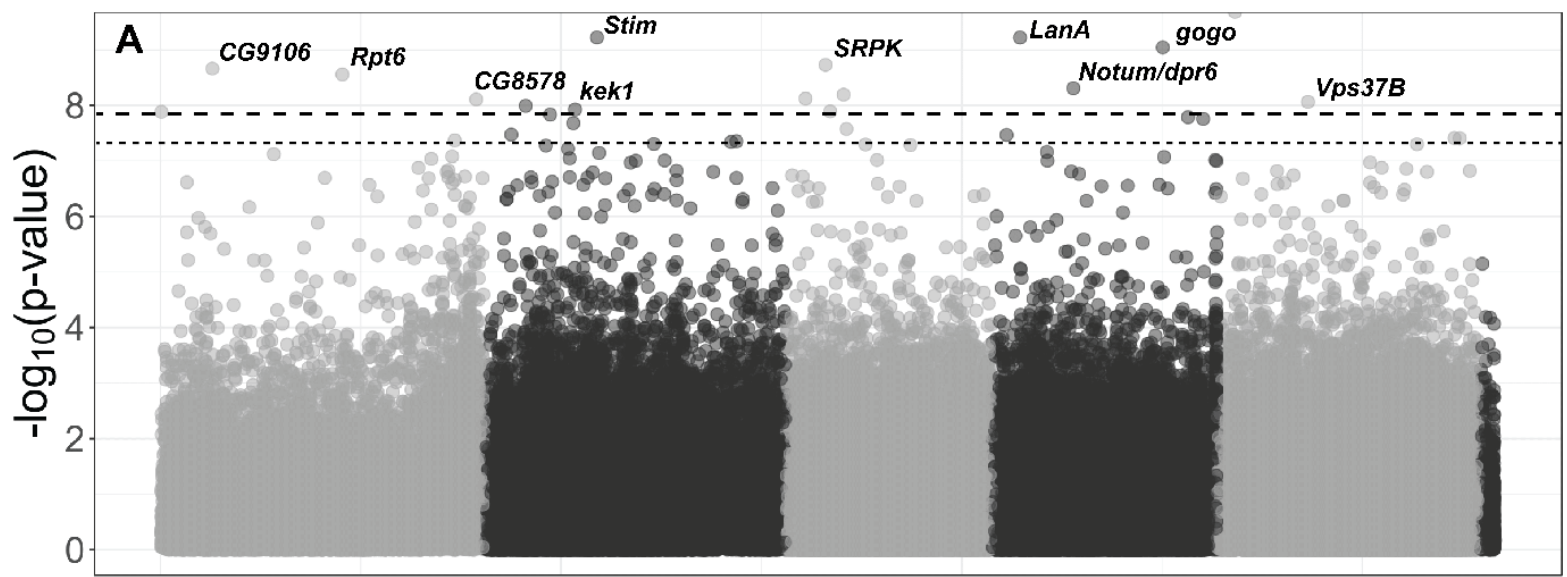

Muller:

A

B C

D

E F
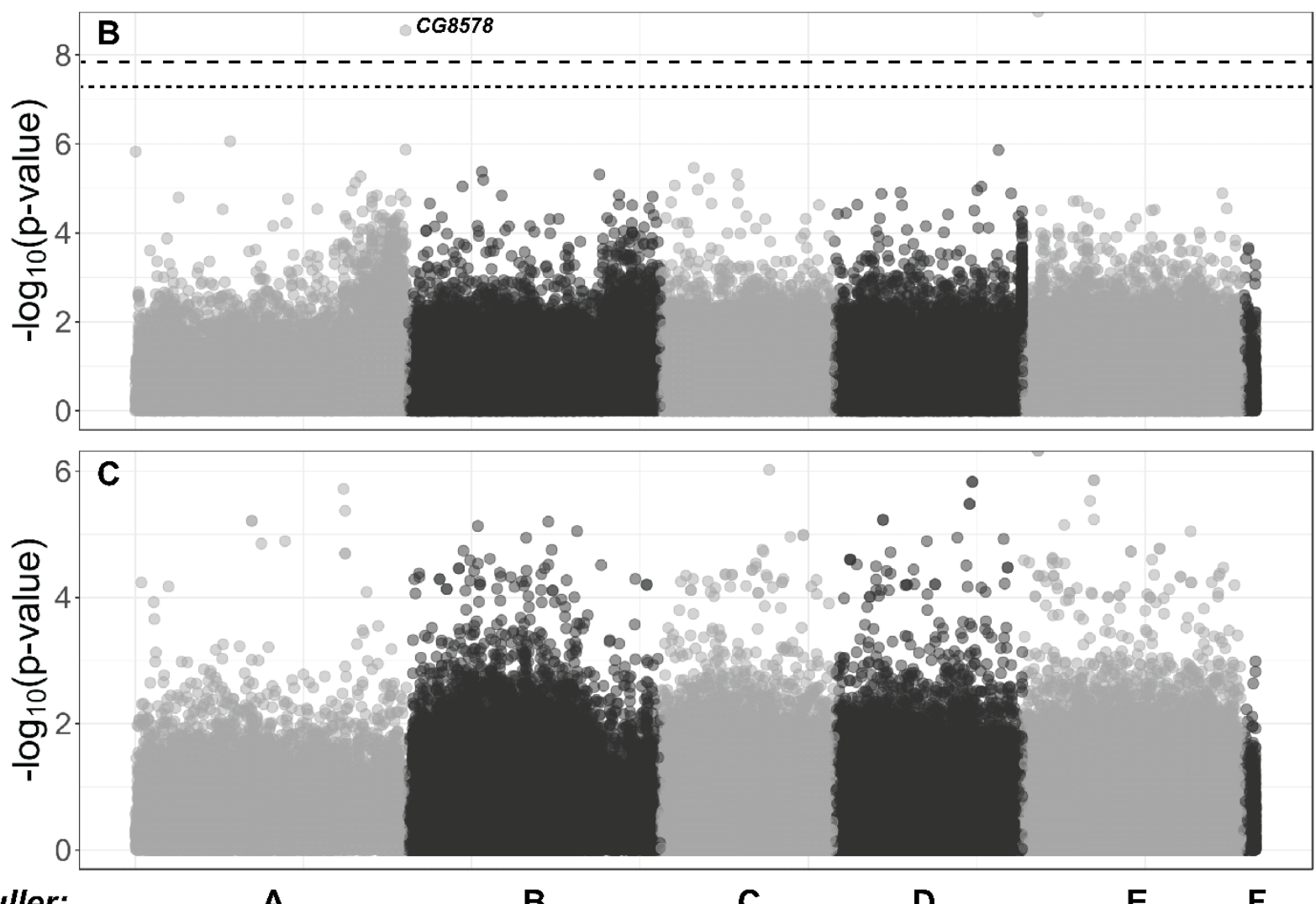
852 Supplementary Figure 11: Effective population size backwards for each population of DiNV going

853 backwards in time, estimated using StairwayPlot. Dotted lines indicate the error windows for $\mathrm{N}_{\mathrm{e}}$ at a given

854 time point. Lines are colored by population.

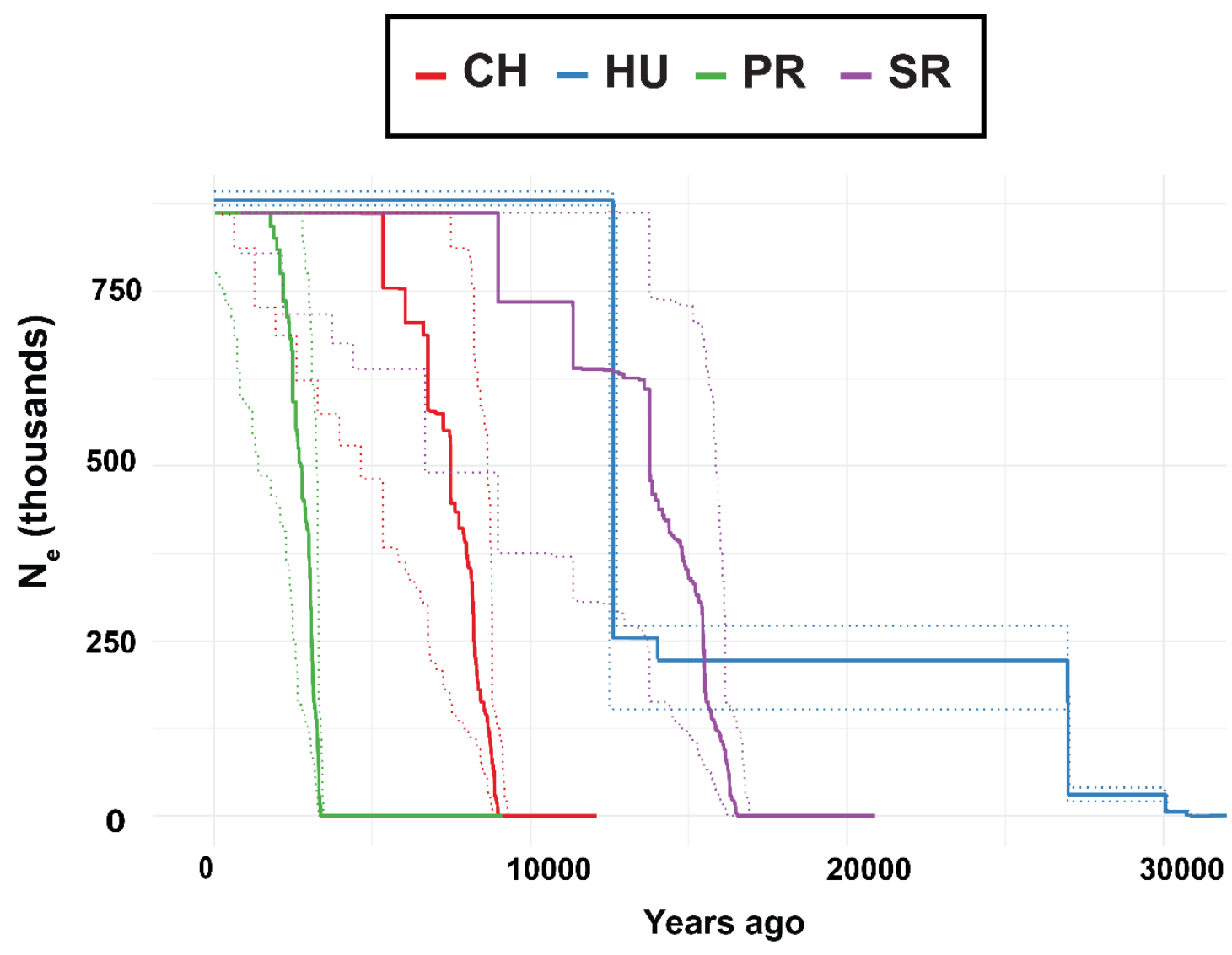

Supplementary Tables

857 Supplementary Table 1: Summary of Drosophila innubila and D. azteca fly samples collected and

858 sequenced for this study, table includes summary of coverage for X chromosome, , Muller B, other

859 autosomes, virus and Wolbachia. Also contains SRA accessions for each strain.

860 Supplementary Table 2: Summary of Drosophila innubila fly RNA and DNA collected and sequenced

861 for this study, including if infected with DiNV.

\section{Supplementary Data}

863 Supplementary Data 1: FPKM of each gene in each sample across the whole $D$. innubila genome,

864 formatted for use in fitting a generalized linear model. Table include the gene name, gene flybase

865 annotation, D. innubila name, if the strains is infected with DiNV and the FPKM.

866 Supplementary Data 2: FPKM of each gene in each sample across the whole D. innubila genome,

867 formatted for differential gene expression analysis. Table include the gene name, gene flybase annotation,

868 D. innubila name, if the strains is infected with DiNV and the FPKM. 
Supplementary Data 3: Differential gene expression analysis results between viral infected and uninfected strains for both D. innubila and D. melanogaster. Genes are labelled as if differentially expressed in one of the two species, or if differentially expressed in both.

872 Supplementary Data 4: VCF file for SNPs in DiNV, used in estimation of population genetic statistics 873 and in GWAS using PLINK.

874 Supplementary Data 5: VCF file for SNPs in D. innubila, used in estimation of population genetic 875 statistics and in GWAS.

876 Supplementary Data 6: Population genetic statistics calculated for each gene in D. innubila using

877 VCFtools for each population.

878 Supplementary Data 7: McDonald-Kreitman statistics calculated for each gene in D. innubila using

879 VCFtools for each population.

880 Supplementary Data 8: Population genetic statistics calculated for each gene in DiNV using VCFtools

881 for each population.

882 Supplementary Data 9: McDonald-Kreitman statistics calculated for each gene in DiNV using VCFtools

883 for each population.

\section{References}

Afonso, C. L., E. R. Tulman, Z. Lu, C. a. Balinsky, B. a. Moser et al., 2001 Genome sequence of a baculovirus pathogenic for Culex nigripalpus. Journal of virology 75: 11157-11165.

Alizon, S., and M. van Baalen, 2008 Transmission-virulence trade-offs in vector-borne diseases. Theor Popul Biol 74: 6-15.

Altschul, S. F., W. Gish, W. Miller, E. W. Myers and D. J. Lipman, 1990 Basic local alignment search tool. Journal of Molecular Biology 215: 403-410.

Anders, S., P. T. Pyl and W. Huber, 2015 HTSeq-A Python framework to work with high-throughput sequencing data. Bioinformatics 31: 166-169.

Anderson, R. M., and R. M. May, 1982 Coevolution of hosts and parasites. Parasitology 85: 411-426.

Argaud, O., L. Croizier, M. López-Ferber and G. Croizier, 1998 Two key mutations in the host-range specificity domain of the p143 gene of Autographa californica nucleopolyhedrovirus are required to kill Bombyx mori larvae. Journal of General Virology 79: 931-935.

Blissard, G. W., and G. F. Rohrmann, 1990 Baculovirus diversity and molecular biology. Annual Review of Entomology 35: 127-155.

Bouckaert, R., J. Heled, D. Kühnert, T. Vaughan, C. H. Wu et al., 2014 BEAST 2: A Software Platform for Bayesian Evolutionary Analysis. PLoS Computational Biology 10: 1-6.

Buffalo, V., 2018 Scythe.

Burgyan, J., and Z. Havelda, 2011 Viral suppressors of RNA silencing. Trends Plant Sci 16: 265-272.

Chateigner, A., A. Bezier, C. Labrousse, D. Jiolle, V. Barbe et al., 2015 Ultra deep sequencing of a baculovirus population reveals widespread genomic variations. Viruses 7: 3625-3646.

Cingolani, P., A. Platts, L. L. Wang, M. Coon, T. Nguyen et al., 2012 A program for annotating and predicting the effects of single nucleotide polymorphisms, SnpEff: SNPs in the genome of Drosophila melanogaster strain w1118; iso-2; iso-3. Fly 6: 80-92.

Coccia, E. M., M. Severa, E. Giacomini, D. Monneron, M. E. Remoli et al., 2004 Viral infection and Tolllike receptor agonists induce a differential expression of type I and lambda interferons in human plasmacytoid and monocyte-derived dendritic cells. European journal of immunology 34: 796-805. 
911

912

913

914

915

916

917

918

919

920

921

922

923

924

925

926

927

928

929

930

931

932

933

934

935

936

937

938

939

940

941

942

943

944

945

946

947

948

949

950

951

952

953

954

955

956

957

958

959

960

961

Costa, A., E. Jan, P. Sarnow and D. Schneider, 2009 The IMD pathway is involved in antiviral immune responses in Drosophila. PLoS ONE 4.

Croizier, G., L. Croizier, O. Argaud and D. Poudevigne, 1994 Extension of Autographa californica nuclear polyhedrosis virus host range by interspecific replacement of a short DNA sequence in the p143 helicase gene. Proceedings of the National Academy of Sciences of the United States of America 91: 48-52.

Daugherty, M. D., and H. S. Malik, 2012 Rules of engagement: molecular insights from host-virus arms races. Annual review of genetics 46: 677-700.

Davey, N. E., G. Trave and T. J. Gibson, 2011 How viruses hijack cell regulation. Trends Biochem Sci 36: 159-169.

Dawkins, R., and J. R. Krebs, 1979 Arms Races between and within Species. Proceedings of the Royal Society of London B: Biological Sciences 205: 489-511.

DePristo, M. A., E. Banks, R. Poplin, K. V. Garimella, J. R. Maguire et al., 2011 A framework for variation discovery and genotyping using next-generation DNA sequencing data. Nature genetics 43: 491498.

Dobzhansky, T., 1937 Genetics and the origin of species. 364.

Dolan, P. T., Z. J. Whitfield and R. Andino, 2018 Mechanisms and Concepts in RNA Virus Population Dynamics and Evolution. Annual Review of Virology 5: 69-92.

Dyer, K. a., and J. Jaenike, 2005 Evolutionary dynamics of a spatially structured host-parasite association: Drosophila innubila and male-killing Wolbachia. Evolution; international journal of organic evolution 59: 1518-1528.

Eilertson, K. E., J. G. Booth and C. D. Bustamante, 2012 SnIPRE: Selection Inference Using a Poisson Random Effects Model. PLoS Computational Biology 8.

Enard, D., L. Cai, C. Gwennap and D. A. Petrov, 2016 Viruses are a dominant driver of protein adaptation in mammals. eLife 5: 1-25.

Feder, A. F., P. S. Pennings, J. Hermisson and D. A. Petrov, 2019 Evolutionary Dynamics in Structured Populations Under Strong Population Genetic Forces. G3 (Bethesda) 9: 3395-3407.

Ferreira, Á. G., H. Naylor, S. S. Esteves, I. S. Pais, N. E. Martins et al., 2014 The Toll-Dorsal Pathway Is Required for Resistance to Viral Oral Infection in Drosophila. PLoS Pathogens 10.

Gavrilets, S., 2003 Perspective: models of speciation: what have we learned in 40 years? Evolution; international journal of organic evolution 57: 2197-2215.

Gavrilets, S., 2004 Fitness Landscapes and the Origin of Species. 117-147.

Ghosh, S., and C. K. Chan, 2016 Analysis of RNA-Seq Data Using TopHat and Cufflinks. Methods Mol Biol 1374: 339-361.

Gifford, R. J., 2012 Viral evolution in deep time: lentiviruses and mammals. Trends Genet 28: 89-100.

Gillespie, J., 2004 Population Genetics: A Concise Guide. 232.

Haas, B. J., A. Papanicolaou, M. Yassour, M. Grabherr, P. D. Blood et al., 2013 De novo transcript sequence reconstruction from RNA-seq using the Trinity platform for reference generation and analysis. Nature protocols 8: 1494-1512.

Hermisson, J., and P. S. Pennings, 2005 Soft sweeps: molecular population genetics of adaptation from standing genetic variation. Genetics 169: 2335-2352.

Hill, T., B. Koseva and R. L. Unckless, 2019 The genome of Drosophila innubila reveals lineage-specific patterns of selection in immune genes. Molecular Biology and Evolution: 1-36.

Hill, T., and R. Unckless, 2020 Selection and demography shape genomic variation in a 'Sky Island' species Biorxiv: 1-35.

Hill, T., and R. L. Unckless, 2017 Baculovirus molecular evolution via gene turnover and recurrent positive selection of key genes. Journal of Virology 91: e01319-01317.

Hill, T., and R. L. Unckless, 2018 The dynamic evolution of Drosophila innubila Nudivirus. Infection, Genetics and Evolution: 1-25.

Hoffmann, J. A., 2003 The immune response of Drosophila. Nature 426: 33-38.

Holmes, E. C., 2007 Viral evolution in the genomic age. PLoS Biol 5: e278. 
962

963

964

965

966

967

968

969

970

971

972

973

974

975

976

977

978

979

980

981

982

983

984

985

986

987

988

989

990

991

992

993

994

995

996

997

998

999

1000

1001

1002

1003

1004

1005

1006

1007

1008

1009

1010

1011

Http://broadinstitute.github.io/picard, Picard.

Hultmark, D., 2003 Drosophila immunity: Paths and patterns. Current Opinion in Immunology 15: 12-19. Jackson, T., 2009 The Use of Oryctes Virus for Control of Rhinoceros Beetle in the Pacific Islands, pp. 133-140 in Use of Microbes for Control and Eradication of Invasive Arthropods SE - 8, edited by A. Hajek, T. Glare and M. O'Callaghan. Springer Netherlands.

Jackson, T. A., A. M. Crawford and T. R. Glare, 2005 Oryctes virus - Time for a new look at a useful biocontrol agent. Journal of Invertebrate Pathology 89: 91-94.

Joshi, N., and J. Fass, 2011 Sickle: A sliding window, adaptive, quality-based trimming tool for fastQ files. 1.33 .

Kaltz, O., and J. A. Shykoff, 1998 Local adaptation in host-parasite systems. Heredity 81: 361-370.

Kassambara, A., M. Kosinski and P. Biecek, 2017 survminer: Drawing Survival Curves using'ggplot2'. R package version 0.31 .

Kelly, D. C., 1982 Baculovirus Replication. Journal of General Virology 63: 1-13.

Kondrashov, A. S., S. Sunyaev and F. A. Kondrashov, 2002 Dobzhansky-Muller incompatibilities in protein evolution. PNAS 99: 14878-14883.

Lamiable, O., C. Kellenberger, C. Kemp, L. Troxler, N. Pelte et al., 2016 Cytokine Diedel and a viral homologue suppress the IMD pathway in Drosophila. Proceedings of the National Academy of Sciences 113: 698-703.

Li, H., and R. Durbin, 2009 Fast and accurate short read alignment with Burrows-Wheeler transform. Bioinformatics (Oxford, England) 25: 1754-1760.

Li, H., B. Handsaker, A. Wysoker, T. Fennell, J. Ruan et al., 2009 The sequence alignment/map format and SAMtools. Bioinformatics (Oxford, England) 25: 2078-2079.

Lipsitch, M., S. Siller and M. Nowak, 1996 The evolution of virulence in pathogens with veritcal and horizontal transmission. Evolution 50: 1729-1741.

Liu, X., and Y.-X. Fu, 2015 Exploring population size changes using SNP frequency spectra. Nature genetics 47: 555-559.

Maeda, S., S. G. Kamita and A. Kondo, 1993 Host range expansion of Autographa californica nuclear polyhedrosis virus (NPV) following recombination of a 0.6-kilobase-pair DNA fragment originating from Bombyx mori NPV. Journal of virology 67: 6234-6238.

Martin, M., 2011 Cutadapt removes adapter sequences from high-throughput sequencing reads. Technical Notes: 1-12.

May, R. M., and M. Nowak, 1995 Coinfection and the Evolution of Parasite Virulence. Proceedings: Biological Sciences 261: 209-215.

McDonald, J. H., and M. Kreitman, 1991 Adaptive protein evolution at the Adh locus in Drosophila. Nature 351: 652-654.

McKenna, A., M. Hanna, E. Banks, A. Sivachenko, K. Cibulskis et al., 2010 The Genome Analysis Toolkit: A MapReduce framework for analyzing next-generation DNA sequencing data. Proceedings of the International Conference on Intellectual Capital, Knowledge Management \& Organizational Learning 20: 1297-1303.

Merkling, S. H., and R. P. van Rij, 2013 Beyond RNAi: Antiviral defense strategies in Drosophila and mosquito. Journal of Insect Physiology 59: 159-170.

Mukherjee, K., H. Campos and B. Kolaczkowski, 2013 Evolution of animal and plant dicers: early parallel duplications and recurrent adaptation of antiviral RNA binding in plants. Mol Biol Evol 30: 627641.

Nielsen, R., 2005 Molecular signatures of natural selection. Annu Rev Genet 39: 197-218.

Nielsen, R., C. Bustamante, A. G. Clark, S. Glanowski, T. B. Sackton et al., 2005 A scan for positively selected genes in the genomes of humans and chimpanzees. PLoS Biol 3: e170.

Obbard, D. J., K. H. J. Gordon, A. H. Buck and F. M. Jiggins, 2009a The evolution of RNAi as a defence against viruses and transposable elements. Philosophical transactions of the Royal Society of London. Series B, Biological sciences 364: 99-115. 
1012

1013

1014

1015

1016

1017

1018

1019

1020

1021

1022

1023

1024

1025

1026

1027

1028

1029

1030

1031

1032

1033

1034

1035

1036

1037

1038

1039

1040

1041

1042

1043

1044

1045

1046

1047

1048

1049

1050

1051

1052

1053

1054

1055

1056

1057

1058

1059

1060

1061

Obbard, D. J., F. M. Jiggins, D. L. Halligan and T. J. Little, 2006 Natural Selection Drives Extremely Rapid Evolution in Antiviral RNAi Genes. Current biology 16: 580-585.

Obbard, D. J., J. J. Welch, K. W. Kim and F. M. Jiggins, 2009b Quantifying adaptive evolution in the Drosophila immune system. PLoS Genetics 5: e1000698.

Orr, H. A., 1995 Population genetics of speciation: the evolution of hybrid incompatibilities. Genetics 139: $1805-1813$.

Orr, H. A., 2004 Dobzhansky, Bateson, and the genetics of speciation. Genetics 168: 1097-1104.

Orr, H. A., and M. Turelli, 2001 The evolution of postzygotic isolation: accumulating Dobzhansky-Muller incompatibilities. Evolution; international journal of organic evolution 55: 1085-1094.

Palmer, W. H., J. D. Hadfield and D. J. Obbard, 2018a RNA Interference Pathways Display High Rates of Adaptive Protein Evolution in Multiple Invertebrates. Genetics: genetics.300567.302017.

Palmer, W. H., J. Joosten, G. J. Overheul, P. W. Jansen, M. Vermeulen et al., 2019 Induction and suppression of NF- $\mathrm{\kappa B}$ signalling by a DNA virus of Drosophila. Journal of Virology 93: e 0144301418.

Palmer, W. H., N. Medd, P. M. Beard and D. J. Obbard, 2018b Isolation of a natural DNA virus of Drosophila melanogaster, and characterisation of host resistance and immune responses. PLoS Pathogens 14: 1-26.

Paradis, E., J. Claude and K. Strimmer, 2004 APE: analyses of phylogenetics and evolution in R language. Bioinformatics 20: 289-290.

Pennings, P. S., 2012 Standing genetic variation and the evolution of drug resistance in HIV. PLoS Computational Biology 8.

Pennings, P. S., S. Kryazhimskiy and J. Wakeley, 2014 Loss and Recovery of Genetic Diversity in Adapting Populations of HIV. PLoS Genetics 10.

Purcell, S., B. Neale, K. Todd-Brown, L. Thomas, M. A. R. Ferreira et al., 2007 PLINK: A Tool Set for Whole-Genome Association and Population-Based Linkage Analyses. The American Journal of Human Genetics 81: 559-575.

Quast, C., E. Pruesse, P. Yilmaz, J. Gerken, T. Schweer et al., 2013 The SILVA ribosomal RNA gene database project: improved data processing and web-based tools. Nucleic Acids Res 41: D590-596.

Robinson, M. D., D. J. McCarthy and G. K. Smyth, 2009 edgeR: A Bioconductor package for differential expression analysis of digital gene expression data. Bioinformatics 26: 139-140.

Rohrmann, G. F., 2013 Baculovirus Molecular Biology. 211.

Sackton, T. B., B. P. Lazzaro, T. A. Schlenke, J. D. Evans, D. Hultmark et al., 2007 Dynamic evolution of the innate immune system in Drosophila. Nature Genetics 39: 1461-1468.

Sagulenko, P., V. Puller and R. A. Neher, 2018 TreeTime: Maximum-likelihood phylodynamic analysis. Virus Evol 4: vex042.

Schulz, M. H., D. R. Zerbino, M. Vingron and E. Birney, 2012 Oases: Robust de novo RNA-seq assembly across the dynamic range of expression levels. Bioinformatics 28: 1086-1092.

Shultz, A., and T. B. Sackton, 2019 Immune genes are hotspots of shared positive selection across birds and mammals. eLife 8: e41815.

Smit, A. F. A., and R. Hubley, 2008 RepeatModeler Open-1.0.

Smit, A. F. A., and R. Hubley, 2013-2015 RepeatMasker Open-4.0, pp. RepeatMasker.

Soetaert, K., T. Petzoldt and R. W. Setzer, 2010 Solving Differential Equations in R: Package deSolve. Journal of Statistical Software 33: 1-24.

Stoletzki, N., and A. Eyre-Walker, 2011 Estimation of the neutrality index. Molecular Biology and Evolution 28: 63-70.

Team, R. C., 2013 R: A Language and Environment for Statistical Computing, pp. R Foundation for Statistical Computing, Vienna, Austria.

Unckless, R. L., 2011 A DNA Virus of Drosophila. PLoS ONE 6: e26564.

Wang, X.-H. X.-H., R. Aliyari, W.-X. Li, H.-W. Li, K. Kim et al., 2006 RNA interference directs innate immunity against viruses in adult Drosophila. Science 312: 452-454. 
1062

1063

1064

1065

1066

1067

1068

1069

1070

1071

1072

1073

1074

1075

1076

1077

1078

1079

1080

1081

1082

1083

1084

1085

Wang, Y., and J. a. Jehle, 2009 Nudiviruses and other large, double-stranded circular DNA viruses of invertebrates: new insights on an old topic. Journal of invertebrate pathology 101: 187-193.

Wang, Y., R. G. Kleespies, M. B. Ramle and J. A. Jehle, 2008 Sequencing of the large dsDNA genome of Oryctes rhinoceros nudivirus using multiple displacement amplification of nanogram amounts of virus DNA. Journal of Virological Methods 152: 106-108.

Webster, C. L., F. M. Waldron, S. Robertson, D. Crowson, G. Ferrari et al., 2015 The discovery, distribution, and evolution of viruses associated with Drosophila melanogaster. PLoS biology 13: e1002210.

West, C., and N. Silverman, 2018 p38b and JAK-STAT signaling protect against invertebrate iridescent virus 6 infection in Drosophila. Plant Pathology: 1-22.

Williams, G. C., and R. M. Nesse, 1991 The Dawn of Darwinian Medicine. The Quarterly Review of Biology 66: 1-22.

Wilm, A., P. Poh, K. Aw, D. Bertrand, G. Hui et al., 2012 LoFreq: a sequence-quality aware, ultra-sensitive variant caller for uncovering cell-population heterogeneity from high-throughput sequencing datasets. Nucleic Acids Research 40: 11189-11201.

Wilson, B. A., N. R. Garud, A. F. Feder, Z. J. Assaf and P. S. Pennings, 2016 The population genetics of drug resistance evolution in natural populations of viral, bacterial and eukaryotic pathogens. Mol Ecol 25: 42-66.

$\mathrm{Wu}, \mathrm{T}$. D., and S. Nacu, 2010 Fast and SNP-tolerant detection of complex variants and splicing in short reads. Bioinformatics (Oxford, England) 26: 873-881.

Zambon, R. A., M. Nandakumar, V. N. Vakharia and L. P. Wu, 2005 The Toll pathway is important for an antiviral response in Drosophila. Proceedings of the National Academy of Sciences 102: 72577262 . 